Check for updates

Cite this: RSC Adv., 2018, 8, 7385

Received 23rd January 2018

Accepted 9th February 2018

DOI: $10.1039 / \mathrm{c} 8 \mathrm{ra00702k}$

rsc.li/rsc-advances

\title{
Efficient synthesis of ether phosphonates using trichloroacetimidate and acetate coupling methods
}

\author{
Walid. Fathalla, (D) *a Ibrahim. A. I. Ali ${ }^{\text {b }}$ and Pavel. Pazdera ${ }^{c}$ \\ A series of ether phosphonates have been prepared by trichloroacetimidate and acetate coupling methods. \\ Trichloroacetimidates or acetates were treated with primary and secondary alcohols as $\mathrm{O}$-nucleophiles in \\ the presence of catalytic TMSOTf to afford 21 examples of diethyl alkyloxy(substitutedphenyl)methyl \\ phosphonates via $\mathrm{C}-\mathrm{O}$ bond formation in $55-90 \%$ yields and short reaction time.
}

\section{Introduction}

Phosphonates are an important class of organic compounds showing remarkable applications as isosteric analogs of natural phosphate esters. ${ }^{1,2}$ Phosphonates are key intermediates in a variety of synthetically important reactions. The reaction of stabilized phosphonate carbanions (ylides) with aldehydes or ketones to afford E-selective alkenes is known as HornerWadsworth-Emmons reaction (a modified Wittig reaction). ${ }^{3-5} \mathrm{~A}$ number of methods have been reported for the preparation of phosphonates. The Michaelis-Arbuzov reaction ${ }^{6,7}$ is an abundant method for the preparation of phosphonates via $\mathrm{P}-\mathrm{C}$ bond formation. It involves the reaction of an aryl/alkyl halide with trialkyl phosphite to give alkyl phosphonate. Hirao method ${ }^{8,9}$ shows the palladium-catalyzed cross-coupling of dialkylphosphite with aromatic electrophiles for the synthesis of arylphosphonates via aryl C-P bond formation. Recently, $\alpha$ functional phosphonates, $\alpha$-aminophosphonates ${ }^{10} \alpha$-acetoxyphosphonates $^{11}$ and $\alpha$-hydroxyphosphonates ${ }^{12-15}$ showed interesting medicinal applications as antiviral agents and attracted the attention of many research groups. Abramov and Pudovik method ${ }^{16,17}$ described the reaction of aldehydes with di and trialkyl phosphite in the presence of a base to afford $\alpha$ hydroxyphosphonates. An efficient use of $\alpha$-hydroxy phosphonates enables a mild process for the preparation of $\alpha$ substituted methyl phosphonates with promising biological activities.

The use of trichloroacetimidate and acetate methods are well recognized in organic and carbohydrate synthesis via $\mathrm{C}-\mathrm{O}$ or $\mathrm{C}-\mathrm{C}$ bond formations. ${ }^{18-21}$ Earlier we described the reaction of $O$-phthalimidomethyl trichloroacetimidate and $O$-diphenylmethyl trichloroacetimidate with C-nucleophiles in the presence of TMSOTf to afford a series of $N$-substituted

${ }^{a}$ Physics and Math. Engineering Dept., Faculty of Engineering, Port-Said University, Port Said, Egypt. E-mail: walid3369@yahoo.com

${ }^{b}$ Department of Chemistry, Faculty of Science, Suez Canal University, Ismailia, Egypt ${ }^{c}$ Centre for Syntheses at Sustainable Conditions and Their Management, Chemistry Dept., Faculty of Science, Masaryk University, Brno, Czech Republic phthalimides ${ }^{20}$ and benzhydryl derivatives. ${ }^{21}$ Also, we showed the preparation of a number of $\mathrm{N}$-protected non proteinogenic $\alpha$-amino acid esters using trichloroacetimidate or acetate coupling methods via $\mathrm{C}-\mathrm{C}$ bond formation from the corresponding methyl 2-benzamido-2-hydroxyacetate and benzyl(methoxycarbonyl)(hydroxy)methylcarbamate substrates and Cnucleophiles. ${ }^{22}$

The strategies of both trichloroacetimidate and acetate methods involve the transformation of the substrate hydroxyl group into an excellent leaving group, such as trichloroacetimidate or acetate by the reaction with trichloroacetonitrile or acetic anhydride, respectively. The successive addition of poor nucleophiles (alcohols) to these active intermediates in the presence of Lewis acid give interesting products via $\mathrm{C}-\mathrm{O}$ bond formations, Fig. 1.

These findings motivated the development of a series of diethyl alkyloxy(substitutedphenyl)methyl phosphonate 6-8(af), 10 and 12 using trichloroacetimidate and acetate coupling methods via $\mathrm{C}-\mathrm{O}$ bond formation.

\section{Results and discussion}

Nucleophilic addition of diethyl phosphite to benzaldehydes 1a-d in $1: 1$ molar ratio using triethylamine as a base gave the diethyl(hydroxy)arylmethyl phosphonates 2a-d in good yields (Scheme 1). The base-catalyzed addition reaction of trichloroacetonitrile to $\boldsymbol{\alpha}$-hydroxyphosphonates $\mathbf{2 a - d}$ hydroxyl group in the presence of DBU gave the desired diethyl 2-(2,2,2-trichloro-1-iminoethoxy)(3,4dimethoxyphenyl)methylphosphonates 3a-d, in $80-94 \%$ yield, Scheme 1. The reactions of $\alpha$-hydroxyphosphonates $2 \mathbf{b}-\mathbf{d}$ with acetic anhydride in the presence of DMAP ( $N, N$-dimethyl aminopyridine) at room temperature afforded diethylmethylcarbonyloxy(4-methoxyphenyl)methylphosphonates $\mathbf{4 b} \mathbf{b}-\mathbf{d}$, Scheme 1.

The structure assignment of $\alpha$-hydroxyphosphonates $\mathbf{2 a - d}$, active intermediates trichloroacetimidate derivatives $\mathbf{3 a - d}$ and acetate derivatives $\mathbf{4 b}-\mathbf{d}$ were based on ${ }^{1} \mathrm{H},{ }^{13} \mathrm{C}$ and ${ }^{31} \mathrm{P}$ NMR spectroscopic and elemental analysis are shown in the experimental part. 


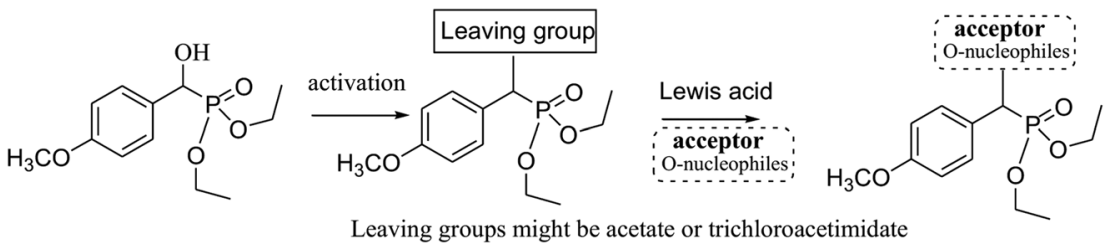

Fig. 1 The trichloroacetimidate and acetate coupling methods from functionalized alcohol substrates.

Both active intermediates trichloroacetimidates $\mathbf{3 a - d}$ and acetates $\mathbf{4 b} \mathbf{b}-\mathbf{d}$ are excellent precursors for the structure modification of the diethyl phosphonate residue via trichloroacetimidate and acetate coupling methods. However, our early results concerning this synthetic method applying diethyl 2-(2,2,2-trichloro-1iminoethoxy)(phenyl)methyl phosphonate (3a) failed to give us the desired products. Thus the reaction of trichloroacetimidate 3a with isobutyl alcohol in the presence of a catalytic amount of TMSOTf at room temperature did not give diethyl 2-methylpropyloxy(phenyl) methyl phosphonate (5a) and instead decomposition was observed and a complex mixture was obtained, Scheme 2 . On the other hand, trichloroacetimidates $\mathbf{3 b} \mathbf{b}-\mathbf{d}$ reacted with a variety of $O$ nucleophile acceptors (alcohols) in the presence of catalytic amount of TMSOTf at room temperature and readily gave a series of functionalized ether phosphonates 6-8(a-f), Scheme 2, Table 1. Similarly, the acetate derivatives $\mathbf{4 b}-\mathbf{d}$ reacted with a variety of $O$ nucleophile acceptors (alcohols) in the presence of a catalytic amount of TMSOTf at room temperature in dichloromethane and readily gave a series of functionalized ether phosphonates 6-8(a-f), Scheme 2, Table 1. A comparative study of both trichloroacetimidate and acetate methodologies was carried out to examine the efficiency of reaction time and \% of yield in both methods. The results showed that, although all compounds were prepared in good yields, there was a slight improvement in the \% of yield and in the reaction time (monitored by TLC) using the trichloroacetimidate method, see Experimental section.
According to the presumed mechanism, the TMSOTf is used to eliminate the trichloroacetimidate and acetate leaving groups with the subsequent formation of carbocation. This carbocation could be stabilized by an efficient electron donor and a phenyl group but it is insufficient. In contrast there was good stabilization in the case of a phenyl residue bearing an electron-donating -OR group at the 4-position. The final step is the nucleophilic attack of the weak alcohol nucleophiles at the stabilized carbocation to form the desired products 6-8(a-f) via an overall $\mathrm{S}_{\mathrm{N}} 1$ mechanism. The attempted reaction of diethyl 2methylpropyloxy(4-methoxyphenyl)methyl phosphonate (6a) with isopropyl alcohol under the same reaction conditions, in the presence of a catalytic amount of TMSOTf at room temperature in dichloromethane, failed to give the corresponding isopropyl ether phosphonate derivative $\mathbf{6 b}$ via transetherification, Scheme 2.

Structure assignment of ether derivatives 6-8(a-f) were based on ${ }^{1} \mathrm{H},{ }^{13} \mathrm{C}$ and ${ }^{31} \mathrm{P}-\mathrm{NMR}$ spectral and physicochemical analysis. The ${ }^{1} \mathrm{H}-\mathrm{NMR}$ spectrum of $\mathbf{6 a}$ shows an interesting doublet signal at $\delta=4.52 \mathrm{ppm}$ with coupling constant $15.0 \mathrm{~Hz}$ typically associated with CHP. This chemical shift is common for all isolated phosphonate derivatives 6-8(a-f) with chemical shifts ranging from 4.37-4.93 ppm and with coupling constant ranging from 12-18 Hz. The ${ }^{1} \mathrm{H}-\mathrm{NMR}$ spectrum exhibit signals at $\delta 0.90,0.93,1.84-1.97$ and 3.16-3.28 ppm due to two $\mathrm{CH}_{3}, \mathrm{CH}$ and $\mathrm{CH}_{2}$ groups, respectively of the isobutyl-residue and signals at $\delta 1.24-1.28$ and $4.02-4.12 \mathrm{ppm}$

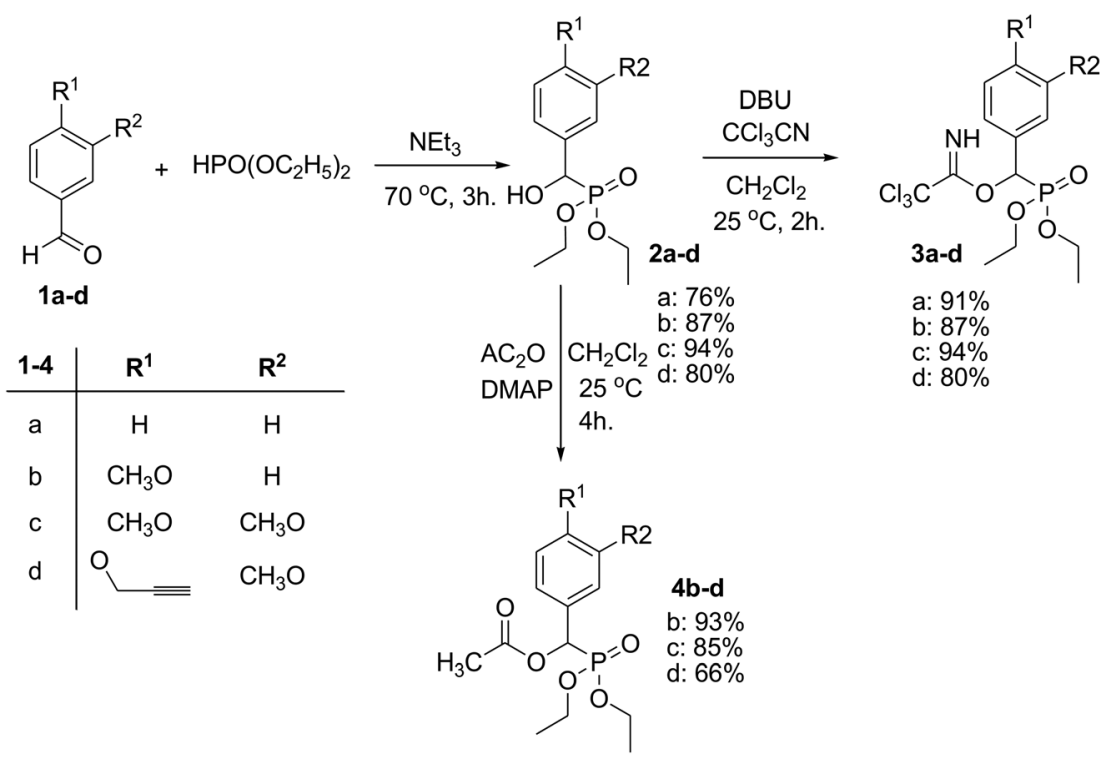

Scheme 1 Synthetic pathway of phosphonates trichloroacetimidates and acetates $3 a-d$ and $4 b-d$. 


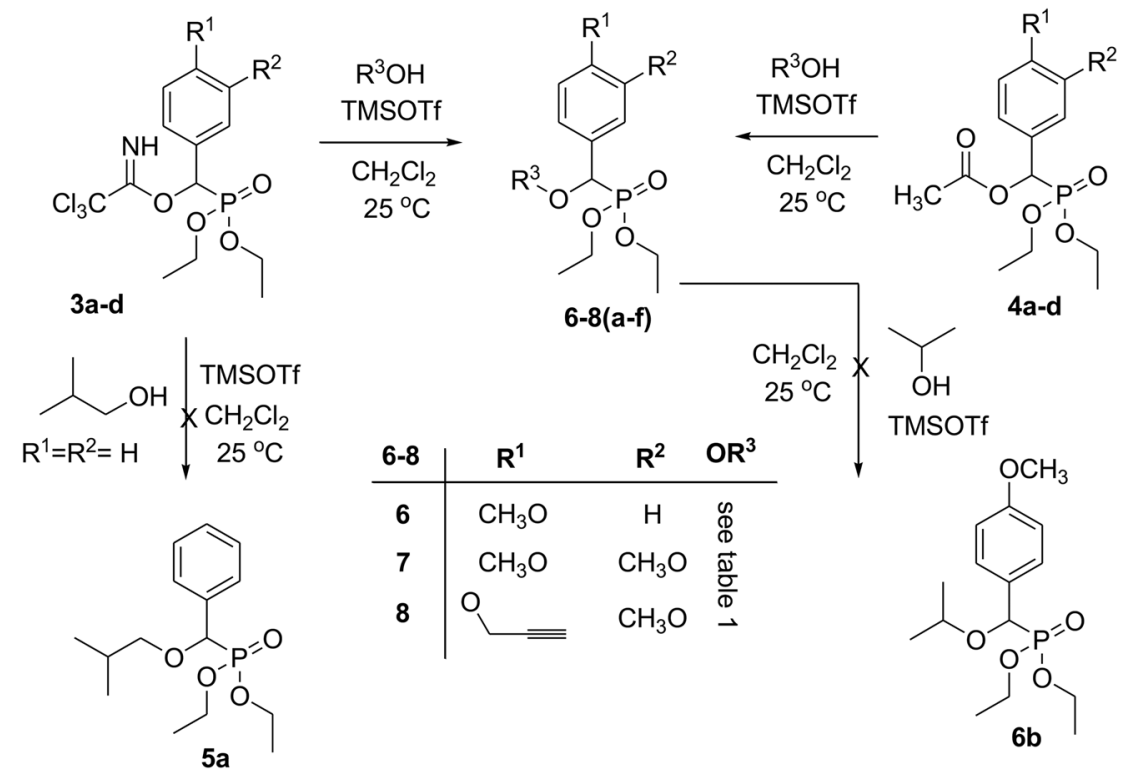

Scheme 2 Synthetic pathway of phosphonates 6-8(a-f) via C-O bond formation.

due to two $\mathrm{OCH}_{2} \mathrm{CH}_{3}$ residue. The ${ }^{13} \mathrm{C}$ NMR spectrum of $6 \mathbf{a}$ shows interesting doublet signals at $\delta 16.3,16.4,62.7,63.0,76.9$ and 77.5 ppm having coupling constants $J_{\mathrm{CP}} 6.0,6.0,6.8,6.8,32.4$ and $169.6 \mathrm{~Hz}$ corresponding to $\mathrm{CH}_{3}, \mathrm{CH}_{3}, \mathrm{OCH}_{2} \mathrm{P}, \mathrm{OCH}_{2} \mathrm{P}, \mathrm{CH}_{2} \mathrm{OCHP}$ and CHP groups, respectively. These signals are common for all isolated products, which confirm the $\mathrm{C}-\mathrm{O}$ bond formation. The previously mentioned signals are good evidences for either the $\mathrm{C}-\mathrm{P}$ coupling, the diastereotopic nature or both for these individual groups. The ${ }^{31} \mathrm{P}-\mathrm{NMR}$ spectrum of $\mathbf{6} \mathbf{a}$ showed a single signal at $\delta=$ $19.5 \mathrm{ppm}$ corresponding to phosphonate phosphorus and was used as an indicator for the purity of the products.

The trichloroacetimidate method proved to be an excellent method for the structure modification of diethyl hydroxy(substituted phenyl)methylphosphonate 2a-d. Next, the use of an interesting derivatized alcohols was explored: (5-methyl-2-phenyl1,3-dioxan-5-yl)methanol 9 and 2 -( $N$-methyl- $N$-phenylamino $)$ ethanol 11. The dioxane derivative 9 was prepared by heating 1,1,1tris(hydroxymethyl)ethane with benzaldehyde in the presence of toluene- $p$-sulfonic acid in benzene and this compound is known to possess potential medicinal chemistry relevance. ${ }^{23}$ Thus the reaction of trichloroacetimidate $\mathbf{3 a} \mathbf{a}-\mathbf{b}$ with dioxane derivative $\mathbf{9}$ in the presence of a catalytic amount of TMSOTf at room temperature in dichloromethane and gave diethyl((5-methyl-2-phenyl-1,3-dioxan5-yl)methoxy)(substitutedphenyl)methyl phosphonate 10a-b in $62-70 \%$ yields, Scheme 3. Also trichloroacetimidate $3 a$ reacted with 2 - $(N$-methyl- $N$-phenylamino)ethanol (11) in the presence of a catalytic amount of TMSOTf at room temperature in dichloromethane and gave diethyl 2-( $N$-methyl- $N$-phenylamino)ethyloxy(4methoxyphenyl)methyl phosphonate 12 in $29 \%$ yield, Scheme 3.

\section{Conclusion}

In conclusion, an efficient and very simple method for the synthesis of various ether phosphonates by trichloroacetimidate and acetate coupling methods is described.
Trichloroacetimidates $\mathbf{3 a}-\mathbf{d}$ or acetates $\mathbf{4 b}$-d were treated with Lewis acid followed by reaction with $O$-nucleophiles to afford the desired products via $\mathrm{C}-\mathrm{O}$ bond formation. Both methods gave good yields of desired products in short reaction times, and generally slightly better results were obtained using the trichloroacetimidate method. Applications of this methodology to synthesize various phosphonate analogues of alcohols containing primary and secondary hydroxy groups as $O$-nucleophiles are under progress in our laboratory.

\section{Experimental}

Solvents were purified and dried in the usual way. The boiling range of the petroleum ether used was $40-60{ }^{\circ} \mathrm{C}$. Thin-layer chromatography (TLC) utilized silica gel 60 F254 plastic plates (E. Merck, layer thickness $0.2 \mathrm{~mm}$ ). Detection was by UV absorption. Melting points were determined on a Buchi 510 melting point apparatus. The ${ }^{1} \mathrm{H},{ }^{13} \mathrm{C}$ and ${ }^{31} \mathrm{P}$ NMR spectra were recorded at $300 \mathrm{MHz}, 75.5$ and 194.4 MHz, respectively (Bruker AC 300) in $\mathrm{CDCl}_{3}$ with tetramethylsilane as an internal standard. The NMR analysis were performed at Organic Chemistry Department Masaryk University, Brno, Czech Republic. Elemental analyses were performed on a Flash EA-1112 instrument at the Microanalytical Laboratory, Faculty of Science, Suez Canal University, Ismailia, Egypt. 3,4-Dimethoxybenzaldehyde 1c and 3-methoxy-4-(prop-2-ynyloxy)benzaldehyde 1d was prepared by alkylation of vaniline as described in the literature. $^{24}$

\section{Diethyl hydroxy(substitutedphenyl)methyl phosphonate $2 \mathrm{a}-\mathrm{d}^{25}$}

To a mixture of (20.7 g, $0.15 \mathrm{~mol})$ of diethyl phosphonate and $1.09 \mathrm{~g}(0.011 \mathrm{~mol})$ of triethylamine was added $(0.15 \mathrm{~mol})$ of aldehydes 1a-d portion wise during a period of $1 \mathrm{~h}$ at $50-70{ }^{\circ} \mathrm{C}$. The reaction mixture was subsequently stirred at $70{ }^{\circ} \mathrm{C}$ for $2 \mathrm{~h}$. After cooling it was left over night to give white crystals, filter 
Table 1 Synthesis of ether phosphonate derivatives $6-8(a-f)$ and the comparative results between trichloroacetimidate and acetate coupling methods $^{a}$

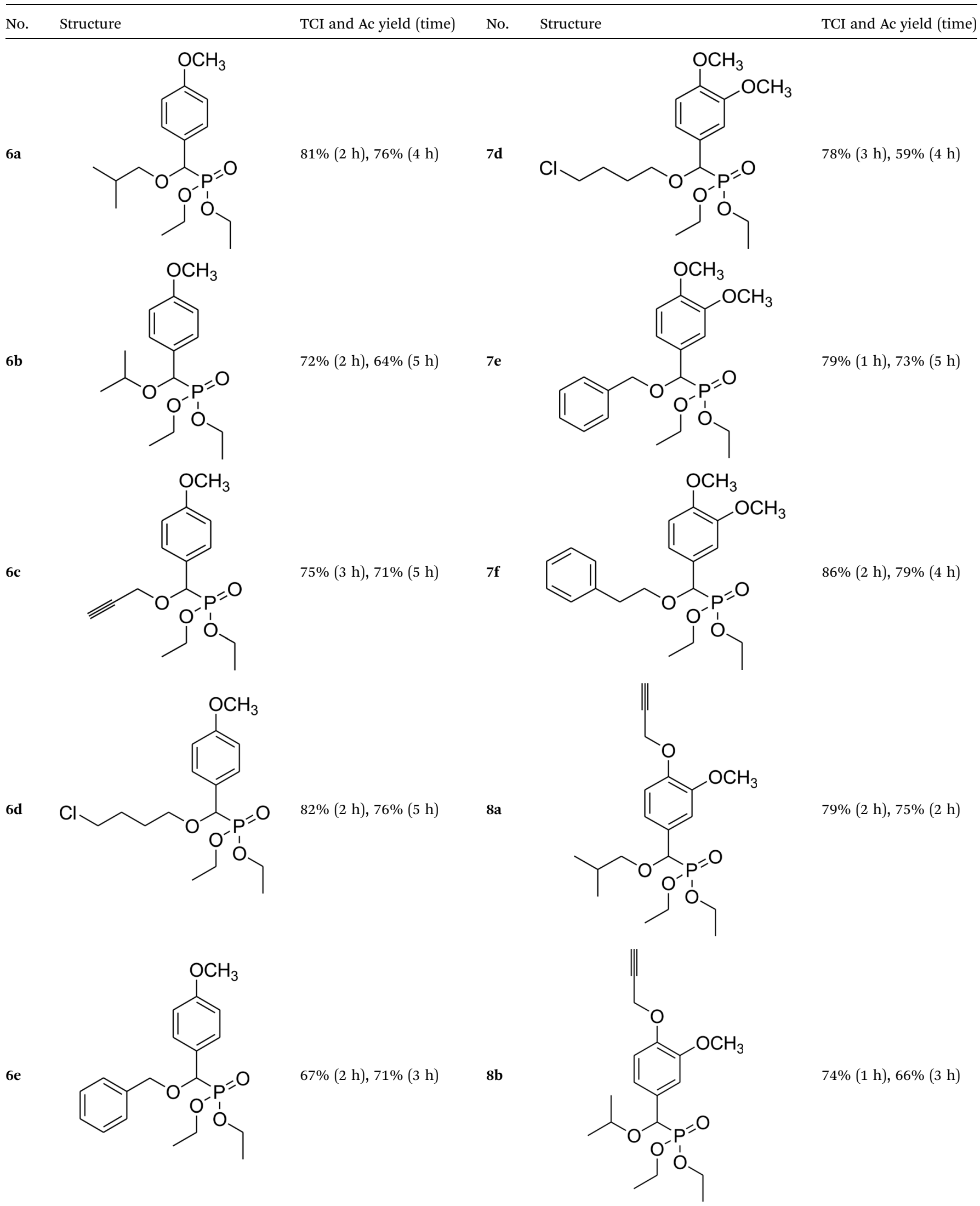


Table 1 (Contd.)

No. Structure TCI and Ac yield (time) No. Structure $\quad$ TCI and Ac yield (time)

6f<smiles>CCOP(=O)(OCC)C(OCCc1ccccc1)c1ccc(OC)cc1</smiles>

$7 a$<smiles>CCOP(=O)(OCC)C(OCC(C)C)c1ccc(OC)c(OC)c1</smiles>

86\% (1 h), 77\% (4 h)

78\% (1 h), 68\% (4 h)<smiles>CCOP(=O)(OCC)C(OC(C)C)c1ccc(OC)c(OC)c1</smiles>

$86 \%(2 \mathrm{~h}), 74 \%(4 \mathrm{~h})$

8f<smiles>C=CC(=C)C(OCCc1ccccc1)P(=O)(OCC)OCC</smiles>

$80 \%(2 \mathrm{~h}), 78 \%(4 \mathrm{~h})$

$81 \%(3 \mathrm{~h}), 72 \%(3 \mathrm{~h})$

$71 \%(2 \mathrm{~h}), 63 \%(3 \mathrm{~h})$<smiles>C=C(C)C(OCc1ccccc1)P(=O)(OCC)OCC</smiles>

$79 \%(2 \mathrm{~h}), 69 \%(4 \mathrm{~h})$

${ }^{a}$ Abbrev. TCI: trichloroacetimidate method, Ac: acetate method. 


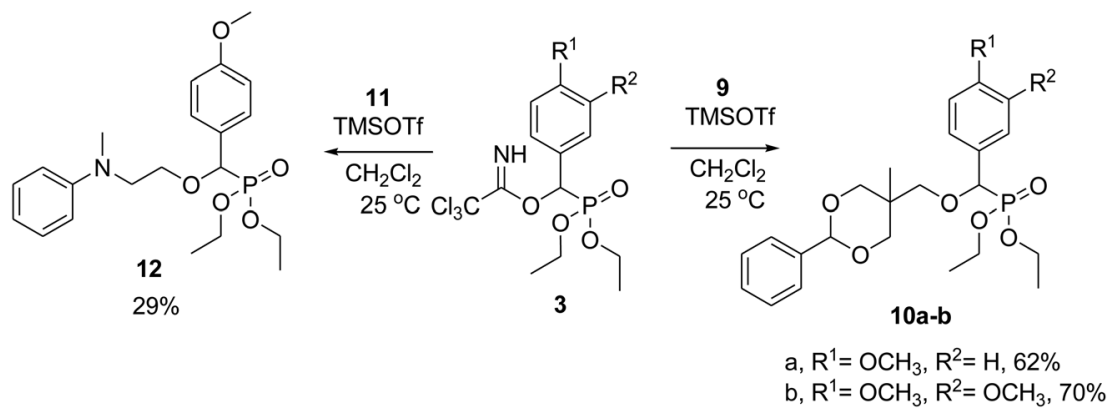

Scheme 3 Synthesis of phosphonates $10 a-b$ and 12 via $\mathrm{C}-\mathrm{O}$ bond formation.

and wash with cold toluene and then recrystallized from toluene to give pure crystals.

\section{Diethyl hydroxy(phenyl)methyl phosphonate (2a)}

$27.5 \mathrm{~g}$, yield $76 \%$ white crystals. Mp 80-81 ${ }^{\circ} \mathrm{C}$. ${ }^{1} \mathrm{H}$ NMR spectrum, $\left(300 \mathrm{MHz}, \mathrm{CDCl}_{3}\right), \delta$, ppm $(J, \mathrm{~Hz}): 1.19-1.34(\mathrm{~m}, 6 \mathrm{H}$, $\left.2 \mathrm{CH}_{3}\right), 3.82\left(\mathrm{~s}, 3 \mathrm{H}, \mathrm{OCH}_{3}\right), 3.91-4.10\left(\mathrm{~m}, 4 \mathrm{H}, 2 \mathrm{CH}_{2}\right), 3.54(\mathrm{bs}, 1 \mathrm{H}$, $\mathrm{OH}), 5.00\left(\mathrm{~d}, J_{\mathrm{HP}}=8.0 \mathrm{~Hz}, 1 \mathrm{H}, \mathrm{CHP}\right), 7.24-7.46(\mathrm{~m}, 5 \mathrm{H}, \mathrm{H}-\mathrm{Ar})$. ${ }^{13} \mathrm{C}-\mathrm{NMR}\left(75.0 \mathrm{MHz}, \mathrm{CDCl}_{3}\right.$ ), $\delta, \mathrm{ppm}: 16.3$ (d, $J_{\mathrm{CP}}=4.0, \mathrm{CH}_{3}$ ), $16.4\left(\mathrm{~d}, J_{\mathrm{CP}}=4.0, \mathrm{CH}_{3}\right), 55.3\left(\mathrm{OCH}_{3}\right), 62.7\left(\mathrm{~d}, J_{\mathrm{CP}}=7.6, \mathrm{CH}_{2} \mathrm{OP}\right)$, $63.4\left(\mathrm{~d}, J_{\mathrm{CP}}=7.6, \mathrm{CH}_{2} \mathrm{OP}\right), 70.2\left(\mathrm{~d}, J_{\mathrm{CP}}=158.4, \mathrm{CHP}\right), 127.1$, 127.2, 128.1, 128.3, 136.8 (C-Ar). ${ }^{31} \mathrm{P}-\mathrm{NMR}$ (194.4 MHz, $\mathrm{CDCl}_{3}$ ), $\delta$, ppm: 21.6 (P). Found, \%: C, 53.89; H, 6.94. For $\mathrm{C}_{11} \mathrm{H}_{17} \mathrm{O}_{4} \mathrm{P}$ (244.1). Calculated, \%: C, 54.10; H, 7.02.

\section{Diethyl hydroxy(4-methoxyphenyl)methyl phosphonate (2b)}

$34.4 \mathrm{~g}$, yield $84 \%$ white crystals. Mp 90-91 ${ }^{\circ} \mathrm{C}$. ${ }^{1} \mathrm{H}-\mathrm{NMR}$ spectrum, (300 MHz, $\left.\mathrm{CDCl}_{3}\right), \delta$, ppm (J, Hz): 1.19-1.29 (m, 6H, $\left.2 \mathrm{CH}_{3}\right), 3.80\left(\mathrm{~s}, 3 \mathrm{H}, \mathrm{OCH}_{3}\right), 3.89-4.10\left(\mathrm{~m}, 4 \mathrm{H}, 2 \mathrm{CH}_{2}\right), 4.33(\mathrm{bs}, 1 \mathrm{H}$, $\mathrm{OH}), 4.95\left(\mathrm{~d}, J_{\mathrm{HP}}=9.0 \mathrm{~Hz}, 1 \mathrm{H}, \mathrm{CHP}\right), 6.87(\mathrm{~d}, J=9.0 \mathrm{~Hz}, 2 \mathrm{H}, \mathrm{H}-$ $\mathrm{Ar}), 7.39-7.43$ (m, 2H, H-Ar). ${ }^{13} \mathrm{C}-\mathrm{NMR}\left(75.0 \mathrm{MHz}, \mathrm{CDCl}_{3}\right.$ ), $\delta$, ppm: $16.3\left(\mathrm{~d}, J_{\mathrm{CP}}=4.0, \mathrm{CH}_{3}\right), 16.4\left(\mathrm{~d}, J_{\mathrm{CP}}=4.0, \mathrm{CH}_{3}\right), 55.2$ $\left(\mathrm{OCH}_{3}\right), 62.9\left(\mathrm{~d}, J_{\mathrm{CP}}=7.6, \mathrm{CH}_{2} \mathrm{OP}\right), 63.2\left(\mathrm{~d}, J_{\mathrm{CP}}=7.6, \mathrm{CH}_{2} \mathrm{OP}\right)$, $70.3\left(\mathrm{~d}, J_{\mathrm{CP}}=161.6\right.$, CHP), 113.0, 113.7, 128.4, 128.5, 128.8, 159.5 (C-Ar). ${ }^{31} \mathrm{P}-\mathrm{NMR}$ (194.4 MHz, $\mathrm{CDCl}_{3}$ ), $\delta, \mathrm{ppm:} 21.7$ (P). Found, \%: C, 52.39; H, 6.81. For $\mathrm{C}_{12} \mathrm{H}_{19} \mathrm{O}_{5} \mathrm{P}$ (274.1). Calculated, $\%:$ C, $52.55 ; \mathrm{H}, 6.98$.

\section{Diethyl hydroxy(3,4-dimethoxyphenyl)methyl phosphonate}

(2c)

$40.6 \mathrm{~g}$, yield $89 \%$ white crystals. Mp 101-103 ${ }^{\circ} \mathrm{C} .{ }^{1} \mathrm{H}-\mathrm{NMR}$ spectrum, (300 MHz, $\left.\mathrm{CDCl}_{3}\right), \delta$, ppm $(J, \mathrm{~Hz}): 1.16-1.26(\mathrm{~m}, 6 \mathrm{H}$, $\left.2 \mathrm{CH}_{3}\right), 3.83\left(\mathrm{~s}, 3 \mathrm{H}, \mathrm{OCH}_{3}\right), 3.88\left(\mathrm{~s}, 3 \mathrm{H}, \mathrm{OCH}_{3}\right), 3.92-4.07(\mathrm{~m}, 4 \mathrm{H}$, $2 \mathrm{CH}_{2}$ ), 4.62 (bs, $\left.1 \mathrm{H}, \mathrm{OH}\right), 4.89$ (d, $\left.J_{\mathrm{HP}}=9.0 \mathrm{~Hz}, 1 \mathrm{H}, \mathrm{CHP}\right), 6.81$ (d, $J=9.0 \mathrm{~Hz}, 1 \mathrm{H}, \mathrm{H}-\mathrm{Ar}), 6.95-7.07$ (m, 1H, H-Ar), 7.08 (s, 1H, HAr). ${ }^{13} \mathrm{C}-\mathrm{NMR}\left(75.0 \mathrm{MHz}, \mathrm{CDCl}_{3}\right), \delta, \mathrm{ppm}: 16.3\left(\mathrm{~d}, J_{\mathrm{CP}}=4.0\right.$, $\left.\mathrm{CH}_{3}\right), 16.4\left(\mathrm{~d}, J_{\mathrm{CP}}=4.0, \mathrm{CH}_{3}\right), 55.8\left(\mathrm{OCH}_{3}\right), 55.9\left(\mathrm{OCH}_{3}\right), 62.9(\mathrm{~d}$, $\left.J_{\mathrm{CP}}=7.6, \mathrm{CH}_{2} \mathrm{OP}\right), 63.2\left(\mathrm{~d}, J_{\mathrm{CP}}=7.6, \mathrm{CH}_{2} \mathrm{OP}\right), 70.4\left(\mathrm{~d}, J_{\mathrm{CP}}=\right.$ 161.6, CHP), 110.5, 110.8, 110.9, 129.3, 148.7, 148.8 (C-Ar). ${ }^{31} \mathrm{P}-$ NMR (194.4 MHz, $\mathrm{CDCl}_{3}$ ), $\delta$, ppm: 21.7 (P). Found, \%: C, 51.12; $\mathrm{H}$, 6.89. For $\mathrm{C}_{13} \mathrm{H}_{21} \mathrm{O}_{6} \mathrm{P}$ (304.1). Calculated, \%: C, 51.31; H, 6.96.
Diethyl hydroxy(3-methoxy-4-(prop-2-yn-1-yloxy)phenyl) methyl phosphonate (2d)

$36.9 \mathrm{~g}$, yield $75 \%$ white crystals. Mp 87-88 ${ }^{\circ} \mathrm{C} .{ }^{1} \mathrm{H}-\mathrm{NMR}$ spectrum, $\left(300 \mathrm{MHz}, \mathrm{CDCl}_{3}\right), \delta, \operatorname{ppm}(J, \mathrm{~Hz}): 1.19-1.37(\mathrm{~m}, 6 \mathrm{H}$, $\left.2 \mathrm{CH}_{3}\right), 2.50(\mathrm{t}, J=3.0 \mathrm{~Hz}, 1 \mathrm{H}, \mathrm{CH}), 3.86\left(\mathrm{~s}, 3 \mathrm{H}, \mathrm{OCH}_{3}\right), 3.92-4.17$ $\left(\mathrm{m}, 4 \mathrm{H}, 2 \mathrm{CH}_{2}\right), 4.39$ (bs, $\left.1 \mathrm{H}, \mathrm{OH}\right), 4.74\left(\mathrm{~d}, J=3.0 \mathrm{~Hz}, 2 \mathrm{H}, \mathrm{OCH}_{2}\right)$, $4.93\left(\mathrm{~d}, J_{\mathrm{HP}}=9.0 \mathrm{~Hz}, 1 \mathrm{H}, \mathrm{CHP}\right), 6.99-7.12(\mathrm{~m}, 3 \mathrm{H}, \mathrm{H}-\mathrm{Ar}) .{ }^{13} \mathrm{C}-$ NMR (75.0 MHz, $\mathrm{CDCl}_{3}$ ), $\delta$, ppm: $16.3\left(\mathrm{~d}, J_{\mathrm{CP}}=4.0, \mathrm{CH}_{3}\right), 16.4$ $\left(\mathrm{d}, J_{\mathrm{CP}}=4.0, \mathrm{CH}_{3}\right), 55.9\left(\mathrm{OCH}_{3}\right), 56.8\left(\mathrm{OCH}_{2}\right), 63.0\left(\mathrm{~d}, J_{\mathrm{CP}}=7.6\right.$, $\mathrm{CH}_{2} \mathrm{OP}$ ), 63.2 (d, $\left.J_{\mathrm{CP}}=7.6, \mathrm{CH}_{2} \mathrm{OP}\right), 70.5\left(\mathrm{~d}, J_{\mathrm{CP}}=160.8, \mathrm{CHP}\right)$, $75.8(\mathrm{C} \equiv \mathrm{CH}), 78.5(\mathrm{C} \equiv \mathrm{CH}), 110.9,114.0,119.5,130.7,146.6$, 149.6 (C-Ar). ${ }^{31} \mathrm{P}-\mathrm{NMR}$ (194.4 MHz, $\mathrm{CDCl}_{3}$ ), $\delta$, ppm: $21.5(\mathrm{P})$. Found, \%: C, 54.68; $\mathrm{H}$, 6.43. For $\mathrm{C}_{15} \mathrm{H}_{21} \mathrm{O}_{6} \mathrm{P}$ (328.1). Calculated, \%: C, 54.88; H, 6.45 .

Preparation of diethyl 2-(2,2,2-trichloro-1iminoethoxy)(substitutedphenyl) methyl phosphonate 3a-d

A stirred solution of diethyl hydroxy(substitutedphenyl)methylphosphonate $\mathbf{2 a - d}(10.0 \mathrm{mmol})$ in dry dichloromethane $(30 \mathrm{~mL})$ was treated with trichloroacetonitrile $(2.9 \mathrm{~mL}, 20 \mathrm{mmol})$ and DBU (0.8 mL, $5.0 \mathrm{mmol})$. The reaction mixture was stirred at room temperature for $2 \mathrm{~h}$. The solvent was evaporated under reduced pressure and the product was purified by column chromatography $5 \%$ triethylamine in dichloromethane, to give 3a-d as whitish oil.

\section{Diethyl 2-(2,2,2-trichloro-1-iminoethoxy)(phenyl)methyl phosphonate (3a)}

$1.92 \mathrm{~g}$, yield 91\% brownish oil. ${ }^{1} \mathrm{H}$ NMR spectrum, $(300 \mathrm{MHz}$, $\left.\mathrm{CDCl}_{3}\right), \delta, \operatorname{ppm}(J, \mathrm{~Hz}): 1.16-1.29\left(\mathrm{~m}, 6 \mathrm{H}, 2 \mathrm{CH}_{3}\right), 3.78(\mathrm{~s}, 3 \mathrm{H}$, $\left.\mathrm{OCH}_{3}\right), 3.94-4.17\left(\mathrm{~m}, 4 \mathrm{H}, 2 \mathrm{CH}_{2}\right), 6.22\left(\mathrm{~d}, J_{\mathrm{HP}}=15.0 \mathrm{~Hz}, 1 \mathrm{H}\right.$, CHP), 6.95-7.53 (m, 5H, H-Ar), 8.55 (brs, 1H, NH). ${ }^{31} \mathrm{P}-\mathrm{NMR}$ (194.4 MHz, $\mathrm{CDCl}_{3}$ ), $\delta$, ppm: 16.4 (P). Found, \%: C, 40.04; H, 4.32; $\mathrm{N}$, 3.48. For $\mathrm{C}_{13} \mathrm{H}_{17} \mathrm{Cl}_{3} \mathrm{NO}_{4} \mathrm{P}$ (387.1). Calculated, \%: C, 40.18; H, 4.41; N, 3.60.

\section{Diethyl(2,2,2-trichloro-1-iminoethoxy)(4-methoxyphenyl) methyl phosphonate (3b)}

$3.6 \mathrm{~g}$, yield $87 \%$ brownish oil. ${ }^{1} \mathrm{H}-\mathrm{NMR}$ spectrum, $(300 \mathrm{MHz}$, $\left.\mathrm{CDCl}_{3}\right), \delta, \operatorname{ppm}(J, \mathrm{~Hz}): 1.20-1.27\left(\mathrm{~m}, 6 \mathrm{H}, 2 \mathrm{CH}_{3}\right), 3.76(\mathrm{~s}, 3 \mathrm{H}$, $\left.\mathrm{OCH}_{3}\right), 3.97-4.16\left(\mathrm{~m}, 4 \mathrm{H}, 2 \mathrm{CH}_{2}\right), 6.20\left(\mathrm{~d}, J_{\mathrm{HP}}=15.0 \mathrm{~Hz}, 1 \mathrm{H}\right.$, CHP), 6.86 (d, $J=9.0 \mathrm{~Hz}, 2 \mathrm{H}, \mathrm{H}-\mathrm{Ar}), 7.44$ (d, $J=6.0 \mathrm{~Hz}, 2 \mathrm{H}, \mathrm{H}-$ 
Ar), 8.51 (brs, $1 \mathrm{H}, \mathrm{NH}) .{ }^{13} \mathrm{C}-\mathrm{NMR}(75.0 \mathrm{MHz}, \mathrm{CDCl} 3), \delta$, ppm: $16.3\left(\mathrm{~d}, J_{\mathrm{CP}}=6.0, \mathrm{CH}_{3}\right), 16.4\left(\mathrm{~d}, J_{\mathrm{CP}}=6.0, \mathrm{CH}_{3}\right), 55.2\left(\mathrm{OCH}_{3}\right)$, $63.3\left(\mathrm{~d}, J_{\mathrm{CP}}=6.0, \mathrm{CH}_{2} \mathrm{OP}\right), 63.5\left(\mathrm{~d}, J_{\mathrm{CP}}=6.0, \mathrm{CH}_{2} \mathrm{OP}\right), 74.8(\mathrm{~d}$, $\left.J_{\mathrm{CP}}=172.1, \mathrm{CHP}\right), 90.9\left(\mathrm{CCl}_{3}\right), 113.8,113.9,124.7,124.7,129.2$, 129.3 (C-Ar), $161.1(\mathrm{C}=\mathrm{N}) .{ }^{31} \mathrm{P}-\mathrm{NMR}$ (194.4 $\mathrm{MHz}, \mathrm{CDCl}_{3}$ ), $\delta$, ppm: 16.5 (P). Found, \%: C, 39.96; H, 4.42; N, 3.29. For $\mathrm{C}_{14}$ $\mathrm{H}_{19} \mathrm{Cl}_{3} \mathrm{NO}_{5} \mathrm{P}$ (417.0). Calculated, \%: C, 40.17; H, 4.57; N, 3.35.

\section{Diethyl(2,2,2-trichloro-1-iminoethoxy)(3,4-dimethoxyphenyl) methyl phosphonate (3c)}

$4.2 \mathrm{~g}$, yield $94 \%$ brownish oil. ${ }^{1} \mathrm{H}-\mathrm{NMR}$ spectrum, $(300 \mathrm{MHz}$, $\left.\mathrm{CDCl}_{3}\right), \delta, \operatorname{ppm}(J, \mathrm{~Hz}): 1.25-1.32\left(\mathrm{~m}, 6 \mathrm{H}, 2 \mathrm{CH}_{3}\right), 3.89(\mathrm{~s}, 6 \mathrm{H}, 2$ $\left.\mathrm{OCH}_{3}\right), 4.02-4.23\left(\mathrm{~m}, 4 \mathrm{H}, 2 \mathrm{CH}_{2}\right), 6.23\left(\mathrm{~d}, J_{\mathrm{HP}}=12.0 \mathrm{~Hz}, 1 \mathrm{H}\right.$, CHP), 6.86 (d, $J=9.0 \mathrm{~Hz}, 1 \mathrm{H}, \mathrm{H}-\mathrm{Ar}), 7.09$ (d, $J=9.0 \mathrm{~Hz}, 2 \mathrm{H}, \mathrm{H}-$ $\mathrm{Ar}$ ), 8.53 (brs, $1 \mathrm{H}, \mathrm{NH}) .{ }^{13} \mathrm{C}-\mathrm{NMR}\left(75.0 \mathrm{MHz}, \mathrm{CDCl}_{3}\right), \delta$, ppm: $16.4\left(\mathrm{~d}, J_{\mathrm{CP}}=6.0, \mathrm{CH}_{3}\right), 16.5\left(\mathrm{~d}, J_{\mathrm{CP}}=6.0, \mathrm{CH}_{3}\right), 55.8\left(\mathrm{OCH}_{3}\right)$, $63.3\left(\mathrm{~d}, J_{\mathrm{CP}}=6.0, \mathrm{CH}_{2} \mathrm{OP}\right), 63.7\left(\mathrm{~d}, J_{\mathrm{CP}}=6.0, \mathrm{CH}_{2} \mathrm{OP}\right), 74.9(\mathrm{~d}$, $\left.J_{\mathrm{CP}}=172.1, \mathrm{CHP}\right), 91.0\left(\mathrm{CCl}_{3}\right), 110.8,120.6,120.7,125.2,148.9$, 149.5 (C-Ar), $161.2(\mathrm{C}=\mathrm{N}) .{ }^{31} \mathrm{P}-\mathrm{NMR}\left(194.4 \mathrm{MHz}, \mathrm{CDCl}_{3}\right)$, $\delta$, ppm: $16.5(\mathrm{P})$. Found, \%: 40.02; $\mathrm{H}, 4.67 ; \mathrm{N}, 3.05$. For $\mathrm{C}_{15^{-}}$ $\mathrm{H}_{21} \mathrm{Cl}_{3} \mathrm{NO}_{6} \mathrm{P}$ (447.0). Calculated, \%: C, 40.15; H, 4.72; N, 3.12.

\section{Diethyl(2,2,2-trichloro-1-iminoethoxy)(3-methoxy-4-(prop-2- yn-1-yloxy)phenyl)methyl phosphonate (3d)}

$3.8 \mathrm{~g}$, yield $80 \%$ brownish oil. ${ }^{1} \mathrm{H}-\mathrm{NMR}$ spectrum, $(300 \mathrm{MHz}$, $\left.\mathrm{CDCl}_{3}\right), \delta, \operatorname{ppm}(J, \mathrm{~Hz}): 1.22\left(\mathrm{~m}, 6 \mathrm{H}, 2 \mathrm{CH}_{3}\right), 2.50(\mathrm{~s}, 1 \mathrm{H}, \mathrm{CH}), 3.85$ $\left(\mathrm{s}, 3 \mathrm{H}, \mathrm{OCH}_{3}\right), 3.99-4.16\left(\mathrm{~m}, 4 \mathrm{H}, \mathrm{CH}, 2 \mathrm{CH}_{2}\right), 4.73(\mathrm{~d}, J=3.0 \mathrm{~Hz}$, $\left.2 \mathrm{H}, \mathrm{OCH}_{2}\right), 6.20\left(\mathrm{~d}, J_{\mathrm{HP}}=12.0 \mathrm{~Hz}, 1 \mathrm{H}, \mathrm{CHP}\right), 6.99-7.28(\mathrm{~m}, 3 \mathrm{H}$, $\mathrm{H}-\mathrm{Ar}$ ), 8.53 (brs, $1 \mathrm{H}, \mathrm{NH}) .{ }^{13} \mathrm{C}-\mathrm{NMR}\left(75.0 \mathrm{MHz}, \mathrm{CDCl}_{3}\right), \delta$, ppm: $16.4\left(\mathrm{~d}, J_{\mathrm{CP}}=6.0, \mathrm{CH}_{3}\right), 16.5\left(\mathrm{~d}, J_{\mathrm{CP}}=6.0, \mathrm{CH}_{3}\right), 55.9\left(\mathrm{OCH}_{3}\right)$, $56.7\left(\mathrm{OCH}_{2}\right), 63.3\left(\mathrm{~d}, J_{\mathrm{CP}}=6.8, \mathrm{CH}_{2} \mathrm{OP}\right), 63.6\left(\mathrm{~d}, J_{\mathrm{CP}}=6.8\right.$, $\left.\mathrm{CH}_{2} \mathrm{OP}\right), 74.1\left(\mathrm{~d}, J_{\mathrm{CP}}=171.4, \mathrm{CHP}\right), 75.9(\mathrm{C} \equiv \mathrm{CH}), 78.4(\mathrm{C} \equiv \mathrm{CH})$, $90.9\left(\mathrm{CCl}_{3}\right), 111.2,113.9,120.3,126.6,147.3,149.6$ (C-Ar), 161.1 $(\mathrm{C}=\mathrm{N}) .{ }^{31} \mathrm{P}-\mathrm{NMR}\left(194.4 \mathrm{MHz}, \mathrm{CDCl}_{3}\right), \delta, \mathrm{ppm}: 16.3(\mathrm{P})$. Found, \%: C, 43.11; $\mathrm{H}, 4.36 ; \mathrm{N}, 2.82$. For $\mathrm{C}_{17} \mathrm{H}_{21} \mathrm{Cl}_{3} \mathrm{NO}_{6} \mathrm{P}$ (471.0). Calculated, \%: C, 43.20; H, 4.48; N, 2.96.

\section{Preparation of diethyl methylcarbonyloxy(substitutedphenyl)} methyl phosphonate $4 \mathrm{~b}-\mathrm{d}$

A solution of diethyl hydroxy(substitutedphenyl)methylphosphonate $2 \mathrm{a}-\mathbf{c}(10.0 \mathrm{mmol})$ in acetic anhydride $(1.2 \mathrm{~mL}$, $12.0 \mathrm{mmol})$ and DMAP $(0.61 \mathrm{~g}, 5.0 \mathrm{mmol})$ and $\mathrm{NEt}_{3}(1.1 \mathrm{~mL}$, $12.0 \mathrm{mmol}$ ) was stirred at room temperature for $4 \mathrm{~h}$. The reaction mixture was evaporated under reduced pressure. The residue was purified by column chromatography $4: 1$ pet. ether/ ethyl-acetate, to give $\mathbf{4 a - c}$ as white oil.

\section{Diethyl methylcarbonyloxy(4-methoxyphenyl)methyl phosphonate (4b)}

$2.94 \mathrm{~g}$, yield 93\% whitish oil. ${ }^{1} \mathrm{H}$ NMR spectrum, $(300 \mathrm{MHz}$, $\left.\mathrm{CDCl}_{3}\right), \delta, \operatorname{ppm}(J, \mathrm{~Hz}): 1.19\left(\mathrm{t}, J=6.0 \mathrm{~Hz}, 3 \mathrm{H}, \mathrm{CH}_{3}\right), 1.28(\mathrm{t}, J=$ $\left.6.0 \mathrm{~Hz}, 3 \mathrm{H}, \mathrm{CH}_{3}\right), 2.13\left(\mathrm{~s}, 3 \mathrm{H}, \mathrm{CH}_{3}\right), 3.79\left(\mathrm{~s}, 3 \mathrm{H}, \mathrm{OCH}_{3}\right), 3.87-4.13$ $\left(\mathrm{m}, 4 \mathrm{H}, 2 \mathrm{CH}_{2}\right), 6.07$ (d, $\left.J_{\mathrm{HP}}=12.0 \mathrm{~Hz}, 1 \mathrm{H}, \mathrm{CHP}\right), 6.87(\mathrm{~d}, J=$ $6.0 \mathrm{~Hz}, 2 \mathrm{H}, \mathrm{H}-\mathrm{Ar}), 7.40-7.44$ (m, 2H, H-Ar). ${ }^{13} \mathrm{C}-\mathrm{NMR}(75.0 \mathrm{MHz}$, $\left.\mathrm{CDCl}_{3}\right), \delta$, ppm: $16.2\left(\mathrm{~d}, J_{\mathrm{CP}}=6.0, \mathrm{CH}_{3}\right), 16.4\left(\mathrm{~d}, J_{\mathrm{CP}}=6.0, \mathrm{CH}_{3}\right)$,
$20.9\left(\mathrm{CH}_{3}\right), 55.2\left(\mathrm{OCH}_{3}\right), 63.1\left(\mathrm{~d}, J_{\mathrm{CP}}=6.8, \mathrm{CH}_{2} \mathrm{OP}\right), 63.6\left(\mathrm{~d}, J_{\mathrm{CP}}=\right.$ 6.8, $\mathrm{CH}_{2} \mathrm{OP}$ ), 70.1 (d, $J_{\mathrm{CP}}=172.9$, CHP), 113.9, 125.5, 129.5, 129.6, 159.9 (C-Ar), 169.3 (CO). ${ }^{31} \mathrm{P}-\mathrm{NMR}$ (194.4 MHz, $\mathrm{CDCl}_{3}$ ), $\delta$, ppm: $18.0(\mathrm{P})$. Found, \%: C, 53.13; H, 6.57. For $\mathrm{C}_{14} \mathrm{H}_{21} \mathrm{O}_{6} \mathrm{P}$ (316.1). Calculated, \%: C, 53.16; H, 6.69.

\section{Diethyl methylcarbonyloxy(3,4-dimethoxyphenyl)methyl phosphonate (4c)}

$2.94 \mathrm{~g}$, yield $85 \%$ whitish oil. ${ }^{1} \mathrm{H}$ NMR spectrum, (300 MHz, $\left.\mathrm{CDCl}_{3}\right), \delta, \operatorname{ppm}(J, \mathrm{~Hz}): 1.15\left(\mathrm{t}, J=6 \mathrm{~Hz}, 3 \mathrm{H}, \mathrm{CH}_{3}\right), 1.24(\mathrm{t}, J=$ $\left.6.0 \mathrm{~Hz}, 3 \mathrm{H}, \mathrm{CH}_{3}\right), 2.10\left(\mathrm{~s}, 3 \mathrm{H}, \mathrm{CH}_{3}\right), 3.82\left(\mathrm{~s}, 3 \mathrm{H}, \mathrm{OCH}_{3}\right), 3.88(\mathrm{~s}$, $\left.3 \mathrm{H}, \mathrm{OCH}_{3}\right), 3.91-4.11\left(\mathrm{~m}, 4 \mathrm{H}, 2 \mathrm{CH}_{2}\right), 6.02\left(\mathrm{~d}, J_{\mathrm{HP}}=12.0 \mathrm{~Hz}, 1 \mathrm{H}\right.$, CHP), 6.79 (d, $J=6.0 \mathrm{~Hz}, 1 \mathrm{H}, \mathrm{H}-\mathrm{Ar}), 6.98-7.02$ (m, 2H, H-Ar). ${ }^{13} \mathrm{C}-\mathrm{NMR}\left(75.0 \mathrm{MHz}, \mathrm{CDCl}_{3}\right.$ ), $\delta, \mathrm{ppm}: 16.3\left(\mathrm{~d}, J_{\mathrm{CP}}=6.0, \mathrm{CH}_{3}\right.$ ), $16.4\left(\mathrm{~d}, J_{\mathrm{CP}}=6.0, \mathrm{CH}_{3}\right), 20.8\left(\mathrm{CH}_{3}\right), 55.8\left(\mathrm{CH}_{3}\right), 55.9\left(\mathrm{CH}_{3}\right), 63.1$ $\left(\mathrm{d}, J_{\mathrm{CP}}=6.8, \mathrm{CH}_{2} \mathrm{OP}\right), 63.7\left(\mathrm{~d}, J_{\mathrm{CP}}=6.8, \mathrm{CH}_{2} \mathrm{OP}\right), 70.3\left(\mathrm{~d}, J_{\mathrm{CP}}=\right.$ 172.9, CHP), 110.9, 120.9, 121.0, 125.7, 148.9, 149.4 (C-Ar), 169.2 (CO). ${ }^{31} \mathrm{P}-\mathrm{NMR}\left(194.4 \mathrm{MHz}, \mathrm{CDCl}_{3}\right.$ ), $\delta$, ppm: 17.9 (P). Found, \%: C, 51.85; H, 6.54. For $\mathrm{C}_{15} \mathrm{H}_{23} \mathrm{O}_{7} \mathrm{P}(346.1)$. Calculated, \%: C, 52.02; H, 6.69.

\section{Diethyl methylcarbonyloxy(3-methoxy-4-propargyloxyphenyl) methyl phosphonate $(4 \mathrm{~d})$}

$2.44 \mathrm{~g}$, yield $66 \%$ whitish oil. ${ }^{1} \mathrm{H}$ NMR spectrum, $(300 \mathrm{MHz}$, $\left.\mathrm{CDCl}_{3}\right), \delta, \operatorname{ppm}(J, \mathrm{~Hz}): 1.13\left(\mathrm{t}, J=6.0 \mathrm{~Hz}, 3 \mathrm{H}, \mathrm{CH}_{3}\right), 1.31(\mathrm{t}, J=$ $\left.6.0 \mathrm{~Hz}, 3 \mathrm{H}, \mathrm{CH}_{3}\right), 2.21\left(\mathrm{~s}, 3 \mathrm{H}, \mathrm{COCH}_{3}\right), 2.51(\mathrm{~s}, 1 \mathrm{H}, \mathrm{CH}), 3.87(\mathrm{~s}$, $\left.3 \mathrm{H}, \mathrm{OCH}_{3}\right), 3.92-4.13\left(\mathrm{~m}, 4 \mathrm{H}, \mathrm{CH}, 2 \mathrm{CH}_{2}\right), 4.74(\mathrm{~d}, J=3.0 \mathrm{~Hz}, 2 \mathrm{H}$, $\mathrm{CH}_{2}$ ), 6.07 (d, $\left.J_{\mathrm{HP}}=12.0 \mathrm{~Hz}, 1 \mathrm{H}, \mathrm{CHP}\right), 6.98-7.08$ (m, 3H, H-Ar). ${ }^{13} \mathrm{C}-\mathrm{NMR}\left(75.0 \mathrm{MHz}, \mathrm{CDCl}_{3}\right.$ ), $\delta, \mathrm{ppm}: 16.3\left(\mathrm{~d}, J_{\mathrm{CP}}=6.0, \mathrm{CH}_{3}\right)$, $16.4\left(\mathrm{~d}, J_{\mathrm{CP}}=6.0, \mathrm{CH}_{3}\right), 20.9\left(\mathrm{CH}_{3}\right), 56.0\left(\mathrm{OCH}_{3}\right), 56.7\left(\mathrm{OCH}_{2}\right)$, $63.2\left(\mathrm{~d}, J_{\mathrm{CP}}=6.0, \mathrm{CH}_{2} \mathrm{OP}\right), 63.3\left(\mathrm{~d}, J_{\mathrm{CP}}=6.0, \mathrm{CH}_{2} \mathrm{OP}\right), 70.1\left(\mathrm{~d}, J_{\mathrm{CP}}\right.$ = 172.9, CHP), $75.9(\mathrm{C} \equiv \mathrm{CH}), 78.4(\mathrm{C} \equiv \mathrm{CH}), 111.9,114.0,120.7$, 127.2, 147.3, 149.6 (C-Ar), 169.2 (CO). ${ }^{31} \mathrm{P}-\mathrm{NMR}$ (194.4 MHz, $\mathrm{CDCl}_{3}$ ), $\delta$, ppm: 17.9 (P). Found, \%: C, 55.04; H, 6.19. For $\mathrm{C}_{17} \mathrm{H}_{23} \mathrm{O}_{7} \mathrm{P}$ (370.1). Calculated, \%: C, 55.13; H, 6.26.

General procedure for the preparation of diethyl alkyloxy(substitutedphenyl)methyl phosphonate 6-8(a-f), 10 and 12

A solution of trichloroacetimidate $\mathbf{3 a}-\mathbf{d}$ or acetate $\mathbf{4 b} \mathbf{b}-\mathbf{d}$ $(1.0 \mathrm{mmol})$ in dichloromethane $(40 \mathrm{~mL})$ was added $O$-nucleophiles (alcohols) $(1.0 \mathrm{mmol})$ and TMSOTf $(0.1 \mathrm{mmol})$. The reaction mixture was stirred at room temperature till completion of the reaction (TLC monitored and the time was recorded). The reaction mixture was neutralized with solid sodium bicarbonate, filtered and concentrated in vacuo. The residue was purified by flash chromatography $1: 2$ pet. ether/ethyl-acetate to give the pure alkyloxy phosphonates 6-8(a-f), 10 and 12 as colorless oil.

\section{Diethyl 2-methylpropyloxy(4-methoxyphenyl)methyl phosphonate (6a)}

$0.26 \mathrm{~g}$, yield 78\% colorless oil. ${ }^{1} \mathrm{H}$ NMR spectrum, $(300 \mathrm{MHz}$, $\left.\mathrm{CDCl}_{3}\right), \delta, \operatorname{ppm}(J, \mathrm{~Hz}): 0.90\left(\mathrm{~d}, J=3.0 \mathrm{~Hz}, 3 \mathrm{H}, \mathrm{CH}_{3}\right), 0.93(\mathrm{~d}, J=$ $\left.3.0 \mathrm{~Hz}, 3 \mathrm{H}, \mathrm{CH}_{3}\right), 1.24-1.28\left(\mathrm{~m}, 6 \mathrm{H}, 2 \mathrm{CH}_{3}\right), 1.84-1.97(\mathrm{~m}, 1 \mathrm{H}$, 
$\mathrm{CH}), 3.16-3.28\left(\mathrm{~m}, 2 \mathrm{H}, \mathrm{CH}_{2}\right), 3.82\left(\mathrm{~s}, 3 \mathrm{H}, \mathrm{OCH}_{3}\right), 4.02-4.12(\mathrm{~m}$, $\left.4 \mathrm{H}, 2 \mathrm{CH}_{2}\right), 4.52\left(\mathrm{~d}, J_{\mathrm{HP}}=15.0 \mathrm{~Hz}, 1 \mathrm{H}, \mathrm{CHP}\right), 6.90(\mathrm{~d}, J=6.0 \mathrm{~Hz}$, 2H, H-Ar), 7.28-7.39 (m, 2H, H-Ar). ${ }^{13} \mathrm{C}-\mathrm{NMR}$ (75.0 MHz, $\mathrm{CDCl}_{3}$ ), $\delta$, ppm: $16.3\left(\mathrm{~d}, J_{\mathrm{CP}}=6.0, \mathrm{CH}_{3}\right), 16.4\left(\mathrm{~d}, J_{\mathrm{CP}}=6.0, \mathrm{CH}_{3}\right), 19.2$ $\left(\mathrm{CH}_{3}\right), 19.3\left(\mathrm{CH}_{3}\right), 28.4(\mathrm{CH}), 55.2\left(\mathrm{OCH}_{3}\right), 62.7\left(\mathrm{~d}, J_{\mathrm{CP}}=6.8\right.$, $\mathrm{CH}_{2} \mathrm{OP}$ ), 63.0 (d, $\left.J_{\mathrm{CP}}=6.8, \mathrm{CH}_{2} \mathrm{OP}\right), 76.9\left(\mathrm{~d}, J_{\mathrm{CP}}=32.4, \mathrm{CH}_{2^{-}}\right.$ OCHP), 77.5 (d, $\left.J_{\mathrm{CP}}=169.6, \mathrm{CHP}\right), 113.7,113.8,127.0,129.2$, 129.3, 159.7 (C-Ar). ${ }^{31} \mathrm{P}-\mathrm{NMR}$ (194.4 $\mathrm{MHz}, \mathrm{CDCl}_{3}$ ), $\delta$, ppm: 19.5 (P). Found, \%: C, 58.04; H, 8.16. For $\mathrm{C}_{16} \mathrm{H}_{27} \mathrm{O}_{5} \mathrm{P}$ (330.2). Calculated, \%: C, 58.17; H, 8.24.

\section{Diethyl 1-methylethyloxy(4-methoxyphenyl)methyl phosphonate (6b)}

$0.26 \mathrm{~g}$, yield $83 \%$ colorless oil. ${ }^{1} \mathrm{H}$ NMR spectrum, $(300 \mathrm{MHz}$, $\left.\mathrm{CDCl}_{3}\right), \delta, \operatorname{ppm}(J, \mathrm{~Hz}): 1.08\left(\mathrm{~d}, J=6.0 \mathrm{~Hz}, 3 \mathrm{H}, \mathrm{CH}_{3}\right), 1.14(\mathrm{~d}, J=$ $\left.6.0 \mathrm{~Hz}, 3 \mathrm{H}, \mathrm{CH}_{3}\right), 1.19-1.25\left(\mathrm{~m}, 6 \mathrm{H}, 2 \mathrm{CH}_{3}\right), 3.57-3.76(\mathrm{~m}, 1 \mathrm{H}$, $\mathrm{CH}), 3.87\left(\mathrm{~s}, 3 \mathrm{H}, \mathrm{OCH}_{3}\right), 3.92-4.09\left(\mathrm{~m}, 4 \mathrm{H}, 2 \mathrm{CH}_{2}\right), 4.64\left(\mathrm{~d}, J_{\mathrm{HP}}=\right.$ $18.0 \mathrm{~Hz}, 1 \mathrm{H}, \mathrm{CHP}), 6.84$ (d, $J=9.0 \mathrm{~Hz}, 2 \mathrm{H}, \mathrm{H}-\mathrm{Ar}), 7.33-7.36$ (m, $2 \mathrm{H}, \mathrm{H}-\mathrm{Ar}$ ). ${ }^{13} \mathrm{C}-\mathrm{NMR}\left(75.0 \mathrm{MHz}, \mathrm{CDCl}_{3}\right), \delta, \mathrm{ppm}: 16.3\left(\mathrm{~d}, J_{\mathrm{CP}}=\right.$ 6.0, $\left.\mathrm{CH}_{3}\right), 16.4\left(\mathrm{~d}, J_{\mathrm{CP}}=6.0, \mathrm{CH}_{3}\right), 20.9\left(\mathrm{CH}_{3}\right), 23.0\left(\mathrm{CH}_{3}\right), 55.1$ $\left(\mathrm{OCH}_{3}\right), 62.7\left(\mathrm{~d}, J_{\mathrm{CP}}=8.0, \mathrm{CH}_{2} \mathrm{OP}\right), 63.1\left(\mathrm{~d}, J_{\mathrm{CP}}=8.0, \mathrm{CH}_{2} \mathrm{OP}\right)$, $71.3\left(\mathrm{~d}, J_{\mathrm{CP}}=13.6, \mathrm{CHOCHP}\right), 75.5\left(\mathrm{~d}, J_{\mathrm{CP}}=171.4, \mathrm{CHP}\right), 113.6$, 113.7, 117.6, 129.3, 159.5, 159.6 (C-Ar). ${ }^{31} \mathrm{P}-\mathrm{NMR}$ (194.4 MHz, $\mathrm{CDCl}_{3}$ ), $\delta$, ppm: 20.0 (P). Found, \%: C, 56.78; H, 7.83. For $\mathrm{C}_{15} \mathrm{H}_{25} \mathrm{O}_{5} \mathrm{P}$ (316.1). Calculated, \%: C, 56.95; H, 7.97.

\section{Diethyl propargyloxy(4-methoxyphenyl)methyl phosphonate (6c)}

$0.19 \mathrm{~g}$, yield $62 \%$ colorless oil. ${ }^{1} \mathrm{H}$ NMR spectrum, $(300 \mathrm{MHz}$, $\left.\mathrm{CDCl}_{3}\right), \delta, \operatorname{ppm}(J, \mathrm{~Hz}): 1.21\left(\mathrm{t}, J=6.0 \mathrm{~Hz}, 3 \mathrm{H}, \mathrm{CH}_{3}\right), 1.30(\mathrm{t}, J=$ $\left.6.0 \mathrm{~Hz}, 3 \mathrm{H}, \mathrm{CH}_{3}\right), 2.46(\mathrm{~s}, 1 \mathrm{H}, \mathrm{CH}), 3.81\left(\mathrm{~s}, 3 \mathrm{H}, \mathrm{OCH}_{3}\right), 3.89-3.95$ $\left(\mathrm{m}, 2 \mathrm{H}, \mathrm{CH}_{2}\right), 4.01-4.08\left(\mathrm{~m}, 4 \mathrm{H}, 2 \mathrm{CH}_{2}\right), 4.93\left(\mathrm{~d}, J_{\mathrm{HP}}=15.0 \mathrm{~Hz}\right.$, 1H, CHP), 6.70-6.91 (m, 4H, H-Ar). ${ }^{13} \mathrm{C}-\mathrm{NMR}$ (75.0 MHz, $\mathrm{CDCl}_{3}$ ), $\delta$, ppm: $16.3\left(\mathrm{~d}, J_{\mathrm{CP}}=6.0, \mathrm{CH}_{3}\right), 16.4\left(\mathrm{~d}, J_{\mathrm{CP}}=6.0, \mathrm{CH}_{3}\right), 55.2$ $\left(\mathrm{OCH}_{3}\right), 56.4\left(\mathrm{~d}, J_{\mathrm{CP}}=15.9, \mathrm{CH}_{2} \mathrm{OCHP}\right), 62.8\left(\mathrm{~d}, J_{\mathrm{CP}}=7.6\right.$, $\mathrm{CH}_{2} \mathrm{OP}$ ), 63.1 (d, $\left.J_{\mathrm{CP}}=7.6, \mathrm{CH}_{2} \mathrm{OP}\right), 75.5(\mathrm{C} \equiv \mathrm{CH}), 75.6\left(\mathrm{~d}, J_{\mathrm{CP}}=\right.$ 172.1, CHP), 78.6 (C $\equiv \mathrm{CH}), 113.9,114.0,125.2,129.8,129.9$, 160.0 (C-Ar). ${ }^{31} \mathrm{P}-\mathrm{NMR}$ (194.4 MHz, $\mathrm{CDCl}_{3}$ ), $\delta, \mathrm{ppm:} 19.2$ (P). Found, \%: C, 57.57; $\mathrm{H}, 6.63$. For $\mathrm{C}_{15} \mathrm{H}_{21} \mathrm{O}_{5} \mathrm{P}$ (312.1). Calculated, $\%:$ C, 57.69; H, 6.78 .

\section{Diethyl 4-chlorobutyloxy(4-methoxyphenyl) methyl phosphonate (6d)}

$0.17 \mathrm{~g}$, yield $46 \%$ colorless oil. ${ }^{1} \mathrm{H}$ NMR spectrum, $(300 \mathrm{MHz}$, $\left.\mathrm{CDCl}_{3}\right), \delta, \operatorname{ppm}(J, \mathrm{~Hz}): 1.17\left(\mathrm{t}, J=6.0 \mathrm{~Hz}, 3 \mathrm{H}, \mathrm{CH}_{3}\right), 1.28(\mathrm{t}, J=$ $\left.6.0 \mathrm{~Hz}, 3 \mathrm{H}, \mathrm{CH}_{3}\right), 1.56-1.92\left(\mathrm{~m}, 4 \mathrm{H}, 2 \mathrm{CH}_{2}\right), 3.38-3.64(\mathrm{~m}, 4 \mathrm{H}$, $\left.2 \mathrm{CH}_{2}\right), 3.83\left(\mathrm{~s}, 3 \mathrm{H}, \mathrm{OCH}_{3}\right), 3.98-4.08\left(\mathrm{~m}, 4 \mathrm{H}, 2 \mathrm{CH}_{2}\right), 4.56\left(\mathrm{~d}, J_{\mathrm{HP}}\right.$ $=12.0 \mathrm{~Hz}, 1 \mathrm{H}, \mathrm{CHP}), 6.84(\mathrm{~d}, J=9.0 \mathrm{~Hz}, 2 \mathrm{H}, \mathrm{H}-\mathrm{Ar}), 7.33-7.23(\mathrm{~m}$, $2 \mathrm{H}, \mathrm{H}-\mathrm{Ar}$ ). ${ }^{13} \mathrm{C}-\mathrm{NMR}\left(75.0 \mathrm{MHz}, \mathrm{CDCl}_{3}\right.$ ), $\delta, \mathrm{ppm}: 16.3$ (d, $J_{\mathrm{CP}}=$ 6.0, $\left.\mathrm{CH}_{3}\right), 16.4\left(\mathrm{~d}, J_{\mathrm{CP}}=6.0, \mathrm{CH}_{3}\right), 26.7\left(\mathrm{CH}_{2}\right), 29.3\left(\mathrm{CH}_{2}\right), 44.9$ $\left(\mathrm{ClCH}_{2}\right), 55.6\left(\mathrm{OCH}_{3}\right), 62.6\left(\mathrm{~d}, J_{\mathrm{CP}}=8.0, \mathrm{CH}_{2} \mathrm{OP}\right), 63.1\left(\mathrm{~d}, J_{\mathrm{CP}}=\right.$ 8.0, $\mathrm{CH}_{2} \mathrm{OP}$ ), 71.6 (d, $\left.J_{\mathrm{CP}}=13.6, \mathrm{CH}_{2} \mathrm{OCHP}\right), 77.5$ (d, $J_{\mathrm{CP}}=172.1$, CHP), 113.6, 114.0, 125.1, 129.7, 129.9, 159.8 (C-Ar). ${ }^{31} \mathrm{P}-\mathrm{NMR}$ (194.4 MHz, $\mathrm{CDCl}_{3}$ ), $\delta$, ppm: 19.1 (P). Found, \%: C, 52.56; H, 7.03. For $\mathrm{C}_{16} \mathrm{H}_{26} \mathrm{ClO}_{5} \mathrm{P}$ (364.1). Calculated, \%: C, 52.68; H, 7.18.
Diethyl benzyloxy(4-methoxyphenyl)methylphosphonate (6e)

$0.33 \mathrm{~g}$, yield 90\% colorless oil. ${ }^{1} \mathrm{H}$ NMR spectrum, $(300 \mathrm{MHz}$, $\left.\mathrm{CDCl}_{3}\right), \delta, \operatorname{ppm}(J, \mathrm{~Hz}): 1.20\left(\mathrm{t}, J=6.0 \mathrm{~Hz}, 3 \mathrm{H}, \mathrm{CH}_{3}\right), 1.27(\mathrm{t}, J=$ $\left.6.0 \mathrm{~Hz}, 3 \mathrm{H}, \mathrm{CH}_{3}\right), 3.82\left(\mathrm{~s}, 3 \mathrm{H}, \mathrm{OCH}_{3}\right), 3.92-4.13\left(\mathrm{~m}, 4 \mathrm{H}, 2 \mathrm{CH}_{2}\right)$, $4.37\left(\mathrm{~d}, J_{\mathrm{HP}}=12.0 \mathrm{~Hz}, 1 \mathrm{H}, \mathrm{CHP}\right), 4.60-4.71\left(\mathrm{~m}, 2 \mathrm{H}, \mathrm{CH}_{2}\right), 6.92(\mathrm{~d}$, $J=6.0 \mathrm{~Hz}, 2 \mathrm{H}, \mathrm{H}-\mathrm{Ar}), 7.31-40$ (m, 7H, H-Ar). ${ }^{13} \mathrm{C}-\mathrm{NMR}(75.0$ $\mathrm{MHz}, \mathrm{CDCl}_{3}$ ), $\delta$, ppm: $16.3\left(\mathrm{~d}, J_{\mathrm{CP}}=6.0, \mathrm{CH}_{3}\right), 16.4\left(\mathrm{~d}, J_{\mathrm{CP}}=6.0\right.$, $\left.\mathrm{CH}_{3}\right), 55.3\left(\mathrm{OCH}_{3}\right), 62.9\left(\mathrm{~d}, J_{\mathrm{CP}}=6.0, \mathrm{CH}_{2} \mathrm{OP}\right), 63.2\left(\mathrm{~d}, J_{\mathrm{CP}}=6.0\right.$, $\mathrm{CH}_{2} \mathrm{OP}$ ), 71.4 (d, $\left.J_{\mathrm{CP}}=15.1, \mathrm{CH}_{2} \mathrm{OCHP}\right), 76.4$ (d, $J_{\mathrm{CP}}=172.9$, CHP), 113.9, 114.0, 126.2, 128.0, 128.2, 128.4, 129.6, 129.7, 137.1, 159.9 (C-Ar). ${ }^{31} \mathrm{P}-\mathrm{NMR}$ (194.4 $\mathrm{MHz}, \mathrm{CDCl}_{3}$ ), $\delta$, ppm: 19.6 (P). Found, \%: C, 62.48; H, 6.76. For $\mathrm{C}_{19} \mathrm{H}_{25} \mathrm{O}_{5} \mathrm{P}$ (364.1). Calculated, \%: C, 62.63; H, 6.92.

\section{Diethyl 2-phenylethyloxy(4-methoxyphenyl)methyl phosphonate (6f)}

$0.15 \mathrm{~g}$, yield $39 \%$ colorless oil. ${ }^{1} \mathrm{H}$ NMR spectrum, $(300 \mathrm{MHz}$, $\left.\mathrm{CDCl}_{3}\right), \delta, \operatorname{ppm}(\mathrm{J}, \mathrm{Hz}): 1.13-1.21\left(\mathrm{~m}, 6 \mathrm{H}, 2 \mathrm{CH}_{3}\right), 2.84-2.89(\mathrm{~m}$, $\left.2 \mathrm{H}, \mathrm{CH}_{2}\right), 3.59-3.69\left(\mathrm{~m}, 2 \mathrm{H}, \mathrm{CH}_{2}\right), 3.73\left(\mathrm{~s}, 3 \mathrm{H}, \mathrm{OCH}_{3}\right), 3.89-4.01$ $\left(\mathrm{m}, 4 \mathrm{H}, 2 \mathrm{CH}_{2}\right), 4.56\left(\mathrm{~d}, J_{\mathrm{HP}}=15.0 \mathrm{~Hz}, 1 \mathrm{H}, \mathrm{CHP}\right), 6.83(\mathrm{~d}, J=$ $9.0 \mathrm{~Hz}, 2 \mathrm{H}, \mathrm{H}-\mathrm{Ar}), 7.14-7.28$ (m, 7H, H-Ar). ${ }^{13} \mathrm{C}-\mathrm{NMR}(75.0 \mathrm{MHz}$, $\mathrm{CDCl}_{3}$ ), $\delta$, ppm: $16.3\left(\mathrm{~d}, J_{\mathrm{CP}}=6.0, \mathrm{CH}_{3}\right), 16.4\left(\mathrm{~d}, J_{\mathrm{CP}}=6.0, \mathrm{CH}_{3}\right)$, $36.2\left(\mathrm{CH}_{2}\right), 55.7\left(\mathrm{OCH}_{3}\right), 62.8\left(\mathrm{~d}, J_{\mathrm{CP}}=8.0, \mathrm{CH}_{2} \mathrm{OP}\right), 63.2\left(\mathrm{~d}, J_{\mathrm{CP}}=\right.$ 8.0, $\mathrm{CH}_{2} \mathrm{OP}$ ), $71.3\left(\mathrm{CH}_{2}\right)\left(\mathrm{d}, J_{\mathrm{CP}}=13.6, \mathrm{CH}_{2} \mathrm{OCHP}\right), 78.4\left(\mathrm{~d}, J_{\mathrm{CP}}=\right.$ 170.6, CHP), 113.7, 113.8, 126.2, 126.5, 128.2, 128.9, 129.3, 129.4, 138.6, 159.7, 159.8, 163.6 (C-Ar). ${ }^{31} \mathrm{P}-\mathrm{NMR}$ (194.4 MHz, $\mathrm{CDCl}_{3}$ ), $\delta$, ppm: 19.1 (P). Found, \%: C, 63.26; H, 7.13. For $\mathrm{C}_{20} \mathrm{H}_{27} \mathrm{O}_{5} \mathrm{P}$ (378.2). Calculated, \%: C, 63.48; H, 7.19.

\section{Diethyl 2-methylpropyloxy(3,4-dimethoxyphenyl)methyl phosphonate (7a)}

$0.23 \mathrm{~g}$, yield $65 \%$ colorless oil. ${ }^{1} \mathrm{H}$ NMR spectrum, $(300 \mathrm{MHz}$, $\left.\mathrm{CDCl}_{3}\right), \delta, \operatorname{ppm}(J, \mathrm{~Hz}): 0.86\left(\mathrm{~d}, J=3.0 \mathrm{~Hz}, 3 \mathrm{H}, \mathrm{CH}_{3}\right), 0.89(\mathrm{~d}, J=$ $\left.3.0 \mathrm{~Hz}, 3 \mathrm{H}, \mathrm{CH}_{3}\right), 1.19-1.23\left(\mathrm{~m}, 6 \mathrm{H}, 2 \mathrm{CH}_{3}\right), 1.80-1.91(\mathrm{~m}, 1 \mathrm{H}$, $\mathrm{CH}), 3.12-3.24\left(\mathrm{~m}, 2 \mathrm{H}, \mathrm{CH}_{2}\right), 3.83\left(\mathrm{~s}, 3 \mathrm{H}, \mathrm{OCH}_{3}\right), 3.85(\mathrm{~s}, 3 \mathrm{H}$, $\left.\mathrm{OCH}_{3}\right), 4.01-4.07\left(\mathrm{~m}, 4 \mathrm{H}, 2 \mathrm{CH}_{2}\right), 4.48\left(\mathrm{~d}, J_{\mathrm{HP}}=18.0 \mathrm{~Hz}, 1 \mathrm{H}\right.$, CHP), 6.79-6.92 (m, 2H, H-Ar), 7.00 (s, 1H, H-Ar). ${ }^{13} \mathrm{C}-\mathrm{NMR}$ (75.0 $\mathrm{MHz}, \mathrm{CDCl}_{3}$ ), $\delta$, ppm: $16.3\left(\mathrm{~d}, J_{\mathrm{CP}}=5.3, \mathrm{CH}_{3}\right), 16.4\left(\mathrm{~d}, J_{\mathrm{CP}}=5.3\right.$, $\left.\mathrm{CH}_{3}\right), 19.1\left(\mathrm{CH}_{3}\right), 19.2\left(\mathrm{CH}_{3}\right), 28.4(\mathrm{CH}), 55.8\left(2 \mathrm{OCH}_{3}\right), 62.7$ (d, $\left.J_{\mathrm{CP}}=6.8, \mathrm{CH}_{2} \mathrm{OP}\right), 63.0\left(\mathrm{~d}, J_{\mathrm{CP}}=6.8, \mathrm{CH}_{2} \mathrm{OP}\right), 78.7\left(\mathrm{~d}, J_{\mathrm{CP}}=\right.$ 160.0, CHP), 76.9 (d, $\left.J_{\mathrm{CP}}=32.5, \mathrm{CH}_{2} \mathrm{OCHP}\right), 110.7,110.8,111.0$, 120.6, 127.4, 149.0 (C-Ar). ${ }^{31} \mathrm{P}-\mathrm{NMR}$ (194.4 $\mathrm{MHz}, \mathrm{CDCl}_{3}$ ), $\delta$, ppm: 19.4 (P). Found, \%: C, 56.64; H, 8.07. For $\mathrm{C}_{17} \mathrm{H}_{29} \mathrm{O}_{6} \mathrm{P}$ (360.2). Calculated, \%: C, 56.66; H, 8.11.

\section{Diethyl 1-methylethyloxy(3,4-dimethoxyphenyl)methyl phosphonate (7b)}

$0.26 \mathrm{~g}$, yield $74 \%$ colorless oil. ${ }^{1} \mathrm{H}$ NMR spectrum, $(300 \mathrm{MHz}$, $\mathrm{CDCl}_{3}$ ), $\delta, \operatorname{ppm}(J, \mathrm{~Hz}): 1.08$ (d, $\left.J=6.0 \mathrm{~Hz}, 6 \mathrm{H}, 2 \mathrm{CH}_{3}\right), 1.11-1.22$ (m, 6H, 2CH $\mathrm{CH}_{3}$, 3.54-3.62 (m, 1H, CH), $3.80\left(\mathrm{~s}, 3 \mathrm{H}, \mathrm{OCH}_{3}\right), 3.88$ $\left(\mathrm{s}, 3 \mathrm{H}, \mathrm{OCH}_{3}\right), 3.92-4.06\left(\mathrm{~m}, 4 \mathrm{H}, 2 \mathrm{CH}_{2}\right), 4.59\left(\mathrm{~d}, J_{\mathrm{HP}}=18.0 \mathrm{~Hz}\right.$, 1H, CHP), 6.76-6.91 (m, 2H, H-Ar), 6.99 (s, 1H, H-Ar). ${ }^{13} \mathrm{C}-\mathrm{NMR}$ (75.0 MHz, $\left.\mathrm{CDCl}_{3}\right), \delta$, ppm: $16.3\left(\mathrm{~d}, J_{\mathrm{CP}}=5.3, \mathrm{CH}_{3}\right), 16.4\left(\mathrm{~d}, J_{\mathrm{CP}}=\right.$ 5.3, $\left.\mathrm{CH}_{3}\right), 21.0\left(\mathrm{CH}_{3}\right), 23.0\left(\mathrm{CH}_{3}\right), 55.7\left(\mathrm{OCH}_{3}\right), 55.8\left(\mathrm{OCH}_{3}\right), 62.8$ $\left(\mathrm{d}, J_{\mathrm{CP}}=6.8, \mathrm{CH}_{2} \mathrm{OP}\right), 63.1\left(\mathrm{~d}, J_{\mathrm{CP}}=6.8, \mathrm{CH}_{2} \mathrm{OP}\right), 71.5(\mathrm{OCH})(\mathrm{d}$, 
$J_{\mathrm{CP}}=13.6$, CHOCHP), $75.6\left(\mathrm{~d}, J_{\mathrm{CP}}=167.6, \mathrm{CHP}\right), 110.7,110.8$, 111.0, 120.4, 128.1, 148.8 (C-Ar). ${ }^{31} \mathrm{P}-\mathrm{NMR}$ (194.4 MHz, $\mathrm{CDCl}_{3}$ ), $\delta$, ppm: 19.8 (P). Found, \%: C, 55.39; H, 7.67. For $\mathrm{C}_{16} \mathrm{H}_{27} \mathrm{O}_{6} \mathrm{P}$ (346.2). Calculated, \%: C, 55.48; H, 7.86.

\section{Diethyl propargyloxy(3,4-dimethoxyphenyl)methyl phosphonate (7c)}

$0.19 \mathrm{~g}$, yield $56 \%$ colorless oil. ${ }^{1} \mathrm{H}$ NMR spectrum, $(300 \mathrm{MHz}$, $\left.\mathrm{CDCl}_{3}\right), \delta, \operatorname{ppm}(J, \mathrm{~Hz}): 1.05\left(\mathrm{t}, J=6.0 \mathrm{~Hz}, 3 \mathrm{H}, \mathrm{CH}_{3}\right), 1.15(\mathrm{t}, J=$ $\left.6.0 \mathrm{~Hz}, 3 \mathrm{H}, \mathrm{CH}_{3}\right), 2.39$ (s, $\left.1 \mathrm{H}, \mathrm{CH}\right), 3.72\left(\mathrm{~s}, 3 \mathrm{H}, \mathrm{OCH}_{3}\right), 3.82(\mathrm{~s}$, $\left.3 \mathrm{H}, \mathrm{OCH}_{3}\right), 3.87-3.94\left(\mathrm{~m}, 2 \mathrm{H}, \mathrm{CH}_{2}\right), 3.99-4.17\left(\mathrm{~m}, 4 \mathrm{H}, 2 \mathrm{CH}_{2}\right)$, $4.75\left(\mathrm{~d}, J_{\mathrm{HP}}=15.0 \mathrm{~Hz}, 1 \mathrm{H}, \mathrm{CHP}\right), 6.70-6.91$ (m, 3H, H-Ar). ${ }^{13} \mathrm{C}-$ NMR (75.0 MHz, $\mathrm{CDCl}_{3}$ ), $\delta$, ppm: $16.2\left(\mathrm{~d}, J_{\mathrm{CP}}=6.0, \mathrm{CH}_{3}\right), 16.3$ $\left(\mathrm{d}, J_{\mathrm{CP}}=6.0, \mathrm{CH}_{3}\right), 55.7\left(\mathrm{OCH}_{3}\right), 55.8\left(\mathrm{OCH}_{3}\right), 56.3\left(\mathrm{~d}, J_{\mathrm{CP}}=15.9\right.$, $\mathrm{CH}_{2} \mathrm{OCHP}$ ), 62.8 (d, $\left.J_{\mathrm{CP}}=6.8, \mathrm{CH}_{2} \mathrm{OP}\right), 63.2\left(\mathrm{~d}, J_{\mathrm{CP}}=6.8\right.$, $\left.\mathrm{CH}_{2} \mathrm{OP}\right), 75.6(\mathrm{C} \equiv \mathrm{CH}), 75.7\left(\mathrm{~d}, J_{\mathrm{CP}}=172.1, \mathrm{CHP}\right), 78.4(\mathrm{C} \equiv \mathrm{CH})$, 110.8, 111.2, 121.3, 125.6, 148.9, 149.4 (C-Ar). ${ }^{31} \mathrm{P}-\mathrm{NMR}$ (194.4 $\mathrm{MHz}, \mathrm{CDCl}_{3}$ ), $\delta$, ppm: 19.0 (P). Found, \%: C, 55.95; H, 6.60. For $\mathrm{C}_{16} \mathrm{H}_{23} \mathrm{O}_{6} \mathrm{P}$ (342.1). Calculated, \%: C, 56.14; H, 6.77.

\section{Diethyl 4-chlorobutyloxy(3,4-dimethoxyphenyl)methyl phosphonate (7d)}

$0.27 \mathrm{~g}$, yield $69 \%$ colorless oil. ${ }^{1} \mathrm{H}$ NMR spectrum, $(300 \mathrm{MHz}$, $\left.\mathrm{CDCl}_{3}\right), \delta, \operatorname{ppm}(J, \mathrm{~Hz}): 1.19\left(\mathrm{t}, J=6.0 \mathrm{~Hz}, 3 \mathrm{H}, \mathrm{CH}_{3}\right), 1.34(\mathrm{t}, J=$ $\left.6.0 \mathrm{~Hz}, 3 \mathrm{H}, \mathrm{CH}_{3}\right), 1.50-1.72\left(\mathrm{~m}, 4 \mathrm{H}, 2 \mathrm{CH}_{2}\right), 3.51-3.70(\mathrm{~m}, 2 \mathrm{H}$, $\left.\mathrm{CH}_{2}\right), 3.88\left(\mathrm{~s}, 3 \mathrm{H}, \mathrm{OCH}_{3}\right), 3.90\left(\mathrm{~s}, 3 \mathrm{H}, \mathrm{OCH}_{3}\right), 4.02-4.10(\mathrm{~m}, 2 \mathrm{H}$, $\left.\mathrm{CH}_{2}\right), 4.53\left(\mathrm{~d}, J_{\mathrm{HP}}=15.0 \mathrm{~Hz}, 1 \mathrm{H}, \mathrm{CHP}\right), 6.71-6.87$ (m, 2H, H-Ar), 7.63 (s, $1 \mathrm{H}, \mathrm{H}-\mathrm{Ar}) .{ }^{13} \mathrm{C}-\mathrm{NMR}\left(75.0 \mathrm{MHz}, \mathrm{CDCl}_{3}\right.$ ), $\delta$, ppm: 16.3 (d, $\left.J_{\mathrm{CP}}=6.0, \mathrm{CH}_{3}\right), 16.4\left(\mathrm{~d}, J_{\mathrm{CP}}=6.0, \mathrm{CH}_{3}\right), 27.3\left(\mathrm{CH}_{2}\right), 29.3\left(\mathrm{CH}_{2}\right)$, $44.7\left(\mathrm{ClCH}_{2}\right), 55.4\left(\mathrm{OCH}_{3}\right), 56.9\left(\mathrm{OCH}_{3}\right), 62.6\left(\mathrm{~d}, J_{\mathrm{CP}}=6.8\right.$, $\mathrm{CH}_{2} \mathrm{OP}$ ), $63.5\left(\mathrm{~d}, J_{\mathrm{CP}}=6.8, \mathrm{CH}_{2} \mathrm{OP}\right), 71.4\left(\mathrm{~d}, J_{\mathrm{CP}}=13.6, \mathrm{CH}_{2^{-}}\right.$ OCHP), 78.1 (d, $\left.J_{\mathrm{CP}}=170.6, \mathrm{CHP}\right), 110.5,112.0,121.8,126.4$, 149.0, 149.7 (C-Ar). ${ }^{31} \mathrm{P}-\mathrm{NMR}$ (194.4 MHz, $\mathrm{CDCl}_{3}$ ), $\delta$, ppm: 19.2 (P). Found, \%: C, 51.56; H, 7.01. For $\mathrm{C}_{17} \mathrm{H}_{28} \mathrm{ClO}_{6} \mathrm{P}$ (394.1). Calculated, \%: C, 51.71; H, 7.15.

\section{Diethyl benzyloxy(3,4-dimethoxyphenyl)methyl phosphonate} (7e)

$0.32 \mathrm{~g}$, yield $81 \%$ colorless oil. ${ }^{1} \mathrm{H}$ NMR spectrum, $(300 \mathrm{MHz}$, $\left.\mathrm{CDCl}_{3}\right), \delta, \operatorname{ppm}(J, \mathrm{~Hz}): 1.17\left(\mathrm{t}, J=6.0 \mathrm{~Hz}, 3 \mathrm{H}, \mathrm{CH}_{3}\right), 1.25(\mathrm{t}, J=$ $\left.6.0 \mathrm{~Hz}, 3 \mathrm{H}, \mathrm{CH}_{3}\right), 3.86\left(\mathrm{~s}, 3 \mathrm{H}, \mathrm{OCH}_{3}\right), 3.99\left(\mathrm{~s}, 3 \mathrm{H}, \mathrm{OCH}_{3}\right), 4.02-$ $4.12\left(\mathrm{~m}, 4 \mathrm{H}, 2 \mathrm{CH}_{2}\right), 4.37$ (d, $\left.J_{\mathrm{HP}}=12.0 \mathrm{~Hz}, 1 \mathrm{H}, \mathrm{CHP}\right), 4.61(\mathrm{dd}, J$ $\left.=9.0,12.0 \mathrm{~Hz}, 2 \mathrm{H}, \mathrm{CH}_{2}\right), 6.83(\mathrm{~d}, J=9.0 \mathrm{~Hz}, 1 \mathrm{H}, \mathrm{H}-\mathrm{Ar}), 6.94-6.96$ (m, 1H, H-Ar), 7.05 (s, 1H, H-Ar), 7.29 (s, 5H, H-Ar). ${ }^{13} \mathrm{C}-\mathrm{NMR}$ (75.0 MHz, $\mathrm{CDCl}_{3}$ ), $\delta$, ppm: $16.3\left(\mathrm{~d}, J_{\mathrm{CP}}=6.0, \mathrm{CH}_{3}\right), 16.4(\mathrm{~d}$, $\left.J_{\mathrm{CP}}=6.0, \mathrm{CH}_{3}\right), 55.8\left(\mathrm{OCH}_{3}\right), 55.9\left(\mathrm{OCH}_{3}\right), 62.7\left(\mathrm{~d}, J_{\mathrm{CP}}=6.8\right.$, $\mathrm{CH}_{2} \mathrm{OP}$ ), $63.2\left(\mathrm{~d}, J_{\mathrm{CP}}=6.8, \mathrm{CH}_{2} \mathrm{OP}\right), 71.4\left(\mathrm{~d}, J_{\mathrm{CP}}=14.3, \mathrm{CH}_{2}-\right.$ OCHP), 76.6 (d, $J_{\mathrm{CP}}=171.4$, CHP), 110.8, 110.9, 111.2, 111.3, 121.1, 121.2, 126.7, 127.9, 128.2, 128.3, 137.1, 149.1 (C-Ar). ${ }^{31} \mathrm{P}-$ NMR (194.4 MHz, $\mathrm{CDCl}_{3}$ ), $\delta$, ppm: 19.5 (P). Found, \%: C, 60.87; $\mathrm{H}$, 6.74. For $\mathrm{C}_{20} \mathrm{H}_{27} \mathrm{O}_{6} \mathrm{P}$ (394.2). Calculated, \%: C, 60.91; H, 6.90.
Diethyl 2-phenylethyloxy(3,4-dimethoxyphenyl)methyl phosphonate (7f)

$0.28 \mathrm{~g}$, yield $69 \%$ colorless oil. ${ }^{1} \mathrm{H}$ NMR spectrum, $(300 \mathrm{MHz}$, $\left.\mathrm{CDCl}_{3}\right), \delta, \operatorname{ppm}(J, \mathrm{~Hz}): 1.21\left(\mathrm{t}, J=6.0 \mathrm{~Hz}, 3 \mathrm{H}, \mathrm{CH}_{3}\right), 1.26(\mathrm{t}, J=$ $\left.6.0 \mathrm{~Hz}, 3 \mathrm{H}, \mathrm{CH}_{3}\right), 2.90\left(\mathrm{t}, J=9.0,6.0 \mathrm{~Hz}, 2 \mathrm{H}, \mathrm{CH}_{2}\right), 3.63-3.79(\mathrm{~m}$, $\left.2 \mathrm{H}, \mathrm{CH}_{2}\right), 3.85\left(\mathrm{~s}, 3 \mathrm{H}, \mathrm{OCH}_{3}\right), 3.90\left(\mathrm{~s}, 3 \mathrm{H}, \mathrm{OCH}_{3}\right), 3.97-4.04(\mathrm{~s}$, $\left.4 \mathrm{H}, 2 \mathrm{CH}_{2}\right), 4.55\left(\mathrm{~d}, J_{\mathrm{HP}}=15.0 \mathrm{~Hz}, 1 \mathrm{H}, \mathrm{CHP}\right), 6.79-6.93(\mathrm{~m}, 3 \mathrm{H}$, $\mathrm{H}-\mathrm{Ar}$ ), 7.14-7.28 (m, 5H, H-Ar). ${ }^{13} \mathrm{C}-\mathrm{NMR}\left(75.0 \mathrm{MHz}, \mathrm{CDCl}_{3}\right.$ ) $\delta$, ppm: $16.3\left(\mathrm{~d}, J_{\mathrm{CP}}=6.0, \mathrm{CH}_{3}\right), 16.4\left(\mathrm{~d}, J_{\mathrm{CP}}=6.0, \mathrm{CH}_{3}\right), 36.2$ $\left(\mathrm{CH}_{2}\right), 55.8\left(\mathrm{OCH}_{3}\right), 55.9\left(\mathrm{OCH}_{3}\right), 62.8\left(\mathrm{~d}, J_{\mathrm{CP}}=6.8, \mathrm{CH}_{2} \mathrm{OP}\right), 63.2$ $\left(\mathrm{d}, J_{\mathrm{CP}}=6.8, \mathrm{CH}_{2} \mathrm{OP}\right), 71.3\left(\mathrm{~d}, J_{\mathrm{CP}}=14.3, \mathrm{CH}_{2} \mathrm{OCHP}\right), 78.6\left(\mathrm{~d}, J_{\mathrm{CP}}\right.$ $=169.9$, CHP), 110.7, 110.8, 110.9, 120.7, 120.8, 126.2, 127.1, 128.2, 128.9, 138.7, 149.0, 149.2 (C-Ar). ${ }^{31} \mathrm{P}-\mathrm{NMR}$ (194.4 MHz, $\left.\mathrm{CDCl}_{3}\right) \delta$, ppm: 19.1 (P). Found, \%: C, 61.52; H, 7.08. For $\mathrm{C}_{21} \mathrm{H}_{29} \mathrm{O}_{6} \mathrm{P}$ (408.2). Calculated, \%: C, 61.76; H, 7.16.

\section{Diethyl 2-methylpropyloxy(3-methoxy-4-propargyloxyphenyl) methyl phosphonate (8a)}

$0.25 \mathrm{~g}$, yield $66 \%$ colorless oil. ${ }^{1} \mathrm{H}$ NMR spectrum, $(300 \mathrm{MHz}$, $\left.\mathrm{CDCl}_{3}\right), \delta, \operatorname{ppm}(J, \mathrm{~Hz}): 0.90\left(\mathrm{t}, J=3.0 \mathrm{~Hz}, 6 \mathrm{H}, 2 \mathrm{CH}_{3}\right), 1.21-1.27$ $\left(\mathrm{m}, 6 \mathrm{H}, 2 \mathrm{CH}_{3}\right), 1.82-1.95(\mathrm{~m}, 1 \mathrm{H}, \mathrm{CH}), 2.50(\mathrm{~d}, J=3.0 \mathrm{~Hz}, 1 \mathrm{H}$, $\mathrm{CH}), 3.15-3.27\left(\mathrm{~m}, 2 \mathrm{H}, \mathrm{CH}_{2}\right), 3.86\left(\mathrm{~s}, 3 \mathrm{H}, \mathrm{OCH}_{3}\right), 3.98-4.09(\mathrm{~m}$, $\left.4 \mathrm{H}, 2 \mathrm{CH}_{2}\right), 4.51\left(\mathrm{~d}, J_{\mathrm{HP}}=15.0 \mathrm{~Hz}, 1 \mathrm{H}, \mathrm{CHP}\right), 4.73(\mathrm{~d}, J=3.0 \mathrm{~Hz}$, 2H, $\mathrm{CH}_{2}$ ), 6.90-7.04 (m, 3H, H-Ar). ${ }^{13} \mathrm{C}-\mathrm{NMR}$ (75.0 MHz, $\mathrm{CDCl}_{3}$ ), $\delta$, ppm: $16.3\left(\mathrm{~d}, J_{\mathrm{CP}}=5.3, \mathrm{CH}_{3}\right), 16.4\left(\mathrm{~d}, J_{\mathrm{CP}}=5.3, \mathrm{CH}_{3}\right), 19.2$ $\left(\mathrm{CH}_{3}\right), 19.3\left(\mathrm{CH}_{3}\right), 28.4(\mathrm{CH}), 55.8\left(\mathrm{OCH}_{3}\right), 56.8\left(\mathrm{OCH}_{2}\right), 62.8(\mathrm{~d}$, $\left.J_{\mathrm{CP}}=6.8, \mathrm{CH}_{2} \mathrm{OP}\right), 63.1\left(\mathrm{~d}, J_{\mathrm{CP}}=6.8, \mathrm{CH}_{2} \mathrm{OP}\right), 75.8(\mathrm{C} \equiv \mathrm{CH}), 76.9$ $\left(\mathrm{d}, J_{\mathrm{CP}}=31.7, \mathrm{CH}_{2} \mathrm{OCHP}\right), 77.7(\mathrm{C} \equiv \mathrm{CH}), 78.6\left(\mathrm{~d}, J_{\mathrm{CP}}=160.6\right.$, CHP), 111.3, 113.9, 120.5, 128.9, 146.8, 149.6 (C-Ar). ${ }^{31} \mathrm{P}-\mathrm{NMR}$ (194.4 MHz, $\mathrm{CDCl}_{3}$ ), $\delta$, ppm: 19.3 (P). Found, \%: C, 59.23; H, 7.46. For $\mathrm{C}_{19} \mathrm{H}_{29} \mathrm{O}_{6} \mathrm{P}$ (384.2). Calculated, \%: C, 59.37; H, 7.60.

\section{Diethyl 1-methylethyloxy(3-methoxy-4-propargyloxyphenyl) methyl phosphonate $(8 b)$}

$0.34 \mathrm{~g}$, yield $89 \%$ colorless oil. ${ }^{1} \mathrm{H}$ NMR spectrum, $(300 \mathrm{MHz}$, $\left.\mathrm{CDCl}_{3}\right), \delta, \operatorname{ppm}(J, \mathrm{~Hz}): 1.17-1.25\left(\mathrm{~m}, 12 \mathrm{H}, 4 \mathrm{CH}_{3}\right), 2.49(\mathrm{~d}, J=$ $3.0 \mathrm{~Hz}, 1 \mathrm{H}, \mathrm{CH}), 3.58-3.66(\mathrm{~m}, 1 \mathrm{H}, \mathrm{CH}), 3.84\left(\mathrm{~s}, 3 \mathrm{H}, \mathrm{OCH}_{3}\right)$, 3.93-4.09 (m, 4H, 2CH $), 4.63\left(\mathrm{~d}, J_{\mathrm{HP}}=18.0 \mathrm{~Hz}, 1 \mathrm{H}, \mathrm{CHP}\right), 4.70$ $\left(\mathrm{d}, J=3.0 \mathrm{~Hz}, 2 \mathrm{H}, \mathrm{CH}_{2}\right), 6.89-7.04(\mathrm{~m}, 3 \mathrm{H}, \mathrm{H}-\mathrm{Ar}) .{ }^{13} \mathrm{C}-\mathrm{NMR}(75.0$ $\mathrm{MHz}, \mathrm{CDCl}_{3}$ ), $\delta$, ppm: $16.3\left(\mathrm{~d}, J_{\mathrm{CP}}=6.0, \mathrm{CH}_{3}\right), 16.4\left(\mathrm{~d}, J_{\mathrm{CP}}=6.0\right.$, $\left.\mathrm{CH}_{3}\right), 21.0\left(\mathrm{CH}_{3}\right), 23.1\left(\mathrm{CH}_{3}\right), 55.9\left(\mathrm{OCH}_{3}\right), 56.8\left(\mathrm{OCH}_{2}\right), 62.8(\mathrm{~d}$, $\left.J_{\mathrm{CP}}=7.8, \mathrm{CH}_{2} \mathrm{OP}\right), 63.3\left(\mathrm{~d}, J_{\mathrm{CP}}=7.8, \mathrm{CH}_{2} \mathrm{OP}\right), 71.6\left(\mathrm{~d}, J_{\mathrm{CP}}=13.6\right.$, CHOCHP), $75.8(\mathrm{C} \equiv \mathrm{CH}), 75.6\left(\mathrm{~d}, J_{\mathrm{CP}}=171.4, \mathrm{CHP}\right), 78.5$ $(\mathrm{C} \equiv \mathrm{CH}), 111.3,111.5,113.9,120.4,129.6,146.8$ (C-Ar). ${ }^{31} \mathrm{P}-\mathrm{NMR}$ (194.4 MHz, $\mathrm{CDCl}_{3}$ ), $\delta$, ppm: 19.8 (P). Found, \%: C, 59.22; H, 7.46. For $\mathrm{C}_{19} \mathrm{H}_{29} \mathrm{O}_{6} \mathrm{P}$ (384.2). Calculated, \%: C, 59.37; H, 7.60.

\section{Diethyl propargyloxy(3-methoxy-4-propargyloxyphenyl)methyl} phosphonate $(8 \mathrm{c})$

$0.27 \mathrm{~g}$, yield $74 \%$ colorless oil. ${ }^{1} \mathrm{H}$ NMR spectrum, $(300 \mathrm{MHz}$, $\left.\mathrm{CDCl}_{3}\right), \delta, \operatorname{ppm}(J, \mathrm{~Hz}): 1.11\left(\mathrm{t}, J=6.0 \mathrm{~Hz}, 3 \mathrm{H}, \mathrm{CH}_{3}\right), 1.20(\mathrm{t}, J=$ $\left.6.0 \mathrm{~Hz}, 3 \mathrm{H}, \mathrm{CH}_{3}\right), 2.42(\mathrm{t}, J=3.0 \mathrm{~Hz}, 1 \mathrm{H}, \mathrm{CH}), 2.47(\mathrm{t}, J=3.0 \mathrm{~Hz}$, $1 \mathrm{H}, \mathrm{CH}), 3.78\left(\mathrm{~s}, 3 \mathrm{H}, \mathrm{OCH}_{3}\right), 3.82-3.94\left(\mathrm{~m}, 2 \mathrm{H}, \mathrm{CH}_{2}\right), 3.98-4.08$ $\left(\mathrm{m}, 4 \mathrm{H}, 2 \mathrm{CH}_{2}\right), 4.65\left(\mathrm{~d}, J=3.0 \mathrm{~Hz}, 2 \mathrm{H}, \mathrm{OCH}_{2}\right), 4.81\left(\mathrm{~d}, J_{\mathrm{HP}}=\right.$ $15.0 \mathrm{~Hz}, 1 \mathrm{H}, \mathrm{CHP}), 6.86-6.98$ (m, 3H, H-Ar). ${ }^{13} \mathrm{C}-\mathrm{NMR}(75.0$ 
$\left.\mathrm{MHz}, \mathrm{CDCl}_{3}\right), \delta$, ppm: $16.2\left(\mathrm{~d}, J_{\mathrm{CP}}=6.0, \mathrm{CH}_{3}\right), 16.4\left(\mathrm{~d}, J_{\mathrm{CP}}=6.0\right.$, $\left.\mathrm{CH}_{3}\right), 55.9\left(\mathrm{OCH}_{3}\right), 56.5\left(\mathrm{~d}, J_{\mathrm{CP}}=15.9, \mathrm{CH}_{2} \mathrm{OCHP}\right), 56.9\left(\mathrm{OCH}_{2}\right)$, $62.9\left(\mathrm{~d}, J_{\mathrm{CP}}=6.8, \mathrm{CH}_{2} \mathrm{OP}\right), 63.2\left(\mathrm{~d}, J_{\mathrm{CP}}=6.8, \mathrm{CH}_{2} \mathrm{OP}\right), 75.7\left(\mathrm{~d}, J_{\mathrm{CP}}\right.$ $=169.1, \mathrm{CHP}), 75.7(\mathrm{C} \equiv \mathrm{CH}), 76.0(\mathrm{C} \equiv \mathrm{CH}), 78.3(\mathrm{C} \equiv \mathrm{CH}), 78.5$ $(\mathrm{C} \equiv \mathrm{CH}), 111.6,113.9,121.1,127.1,147.2,149.7$ (C-Ar). ${ }^{31} \mathrm{P}-\mathrm{NMR}$ (194.4 MHz, $\mathrm{CDCl}_{3}$ ), $\delta$, ppm: 18.9 (P). Found, \%: C, 58.92; H, 6.29. For $\mathrm{C}_{18} \mathrm{H}_{23} \mathrm{O}_{6} \mathrm{P}$ (366.1). Calculated, \%: C, 59.01; H, 6.33.

\section{Diethyl 4-chlorobutyloxy(3-methoxy-4-propargyloxyphenyl) methyl phosphonate (8d)}

$0.26 \mathrm{~g}$, yield $62 \%$ colorless oil. ${ }^{1} \mathrm{H}$ NMR spectrum, $(300 \mathrm{MHz}$, $\left.\mathrm{CDCl}_{3}\right), \delta, \operatorname{ppm}(\mathrm{J}, \mathrm{Hz}): 1.15-1.48\left(\mathrm{~m}, 6 \mathrm{H}, 2 \mathrm{CH}_{3}\right), 1.58-1.89(\mathrm{~m}$, $\left.4 \mathrm{H}, 2 \mathrm{CH}_{2}\right), 2.51(\mathrm{t}, J=3.0 \mathrm{~Hz}, 1 \mathrm{H}, \mathrm{CH}), 3.41-3.61\left(\mathrm{~m}, 4 \mathrm{H}, 2 \mathrm{CH}_{2}\right)$, $3.84\left(\mathrm{~s}, 3 \mathrm{H}, \mathrm{OCH}_{3}\right), 3.99-4.06\left(\mathrm{~m}, 4 \mathrm{H}, 2 \mathrm{CH}_{2}\right), 4.48\left(\mathrm{~d}, J_{\mathrm{HP}}=\right.$ $12.0 \mathrm{~Hz}, 1 \mathrm{H}, \mathrm{CHP}), 4.71$ (d, $\left.J=3.0 \mathrm{~Hz}, 2 \mathrm{H}, \mathrm{OCH}_{2}\right), 6.89-7.13(\mathrm{~m}$, $3 \mathrm{H}, \mathrm{H}-\mathrm{Ar}) .{ }^{13} \mathrm{C}-\mathrm{NMR}\left(75.0 \mathrm{MHz}, \mathrm{CDCl}_{3}\right), \delta, \mathrm{ppm}: 16.3\left(\mathrm{~d}, J_{\mathrm{CP}}=\right.$ 6.0, $\left.\mathrm{CH}_{3}\right), 16.4\left(\mathrm{~d}, J_{\mathrm{CP}}=6.0, \mathrm{CH}_{3}\right), 26.9\left(\mathrm{CH}_{2}\right), 29.3\left(\mathrm{CH}_{2}\right), 44.7$ $\left(\mathrm{CH}_{2}\right), 55.9\left(\mathrm{OCH}_{3}\right), 56.0\left(\mathrm{OCH}_{3}\right), 56.7\left(\mathrm{OCH}_{2}\right), 62.9\left(\mathrm{~d}, J_{\mathrm{CP}}=6.8\right.$, $\mathrm{CH}_{2} \mathrm{OP}$ ), 63.1 (d, $\left.J_{\mathrm{CP}}=6.8, \mathrm{CH}_{2} \mathrm{OP}\right), 69.9\left(\mathrm{~d}, J_{\mathrm{CP}}=13.6, \mathrm{CH}_{2^{-}}\right.$ OCHP), 75.9 (C $\equiv \mathrm{CH}), 78.4\left(\mathrm{~d}, J_{\mathrm{CP}}=170.6, \mathrm{CHP}\right), 78.6(\mathrm{C} \equiv \mathrm{CH})$, 110.8, 112.0, 121.8, 126.4, 149.0, 149.7 (C-Ar). ${ }^{31} \mathrm{P}-\mathrm{NMR}$ (194.4 $\mathrm{MHz}, \mathrm{CDCl}_{3}$ ), $\delta$, ppm: 19.2 (P). ${ }^{31} \mathrm{P}-\mathrm{NMR}$ (194.4 MHz, $\mathrm{CDCl}_{3}$ ), $\delta$, ppm: $19.2(\mathrm{P})$. Found, \%: C, 54.34; $\mathrm{H}, 6.65$. For $\mathrm{C}_{19} \mathrm{H}_{28} \mathrm{ClO}_{6} \mathrm{P}$ (418.1). Calculated, \%: C, 54.48; H, 6.74.

\section{Diethyl benzyloxy(3-methoxy-4-propargyloxyphenyl)methyl phosphonate (8e)}

$0.36 \mathrm{~g}$, yield $87 \%$ colorless oil. ${ }^{1} \mathrm{H}$ NMR spectrum, $(300 \mathrm{MHz}$, $\left.\mathrm{CDCl}_{3}\right), \delta, \operatorname{ppm}(J, \mathrm{~Hz}): 1.19\left(\mathrm{~d}, J=6.0 \mathrm{~Hz}, 3 \mathrm{H}, \mathrm{CH}_{3}\right), 1.24(\mathrm{~d}, J=$ $\left.6.0 \mathrm{~Hz}, 3 \mathrm{H}, \mathrm{CH}_{3}\right), 2.52(\mathrm{~d}, J=3.0 \mathrm{~Hz}, 1 \mathrm{H}, \mathrm{CH}), 3.87(\mathrm{~s}, 3 \mathrm{H}$, $\left.\mathrm{OCH}_{3}\right), 3.90-4.13\left(\mathrm{~m}, 4 \mathrm{H}, 2 \mathrm{CH}_{2}\right), 4.38$ (d, $\left.J=12.0 \mathrm{~Hz}, 1 \mathrm{H}, \mathrm{CH}\right)$, $4.40\left(\mathrm{~d}, J_{\mathrm{HP}}=12.0 \mathrm{~Hz}, 1 \mathrm{H}, \mathrm{CHP}\right), 4.67(\mathrm{~d}, J=12.0 \mathrm{~Hz}, 1 \mathrm{H}, \mathrm{CH})$, 4.77 (d, $J=3.0 \mathrm{~Hz}, 2 \mathrm{H}, \mathrm{CH}_{2}$ ), 6.94-6.98 (m, 3H, H-Ar), 7.06-7.32 (m, 5H, H-Ar). ${ }^{13} \mathrm{C}-\mathrm{NMR}\left(75.0 \mathrm{MHz}, \mathrm{CDCl}_{3}\right.$ ), $\delta$, ppm: 16.3 (d, $J_{\mathrm{CP}}$ $\left.=6.0, \mathrm{CH}_{3}\right), 16.5\left(\mathrm{~d}, J_{\mathrm{CP}}=6.0, \mathrm{CH}_{3}\right), 55.9\left(\mathrm{OCH}_{3}\right), 56.8\left(\mathrm{OCH}_{2}\right)$, $62.8\left(\mathrm{~d}, J_{\mathrm{CP}}=6.8, \mathrm{CH}_{2} \mathrm{OP}\right), 63.1\left(\mathrm{~d}, J_{\mathrm{CP}}=6.8, \mathrm{CH}_{2} \mathrm{OP}\right), 71.5\left(\mathrm{~d}, J_{\mathrm{CP}}\right.$ $\left.=14.3, \mathrm{CH}_{2} \mathrm{OCHP}\right), 75.9(\mathrm{C} \equiv \mathrm{CH}), 76.6\left(\mathrm{~d}, J_{\mathrm{CP}}=170.6, \mathrm{CHP}\right)$, $78.5(\mathrm{C} \equiv \mathrm{CH}), 111.6,111.7,113.9,120.8,120.9,127.9,128.2$, 128.4, 137.1, 147.1, 149.8 (C-Ar), ${ }^{31} \mathrm{P}-\mathrm{NMR}$ (194.4 MHz, $\mathrm{CDCl}_{3}$ ), $\delta$, ppm: 19.4 (P). Found, \%: C, 63.03; H, 6.48. For $\mathrm{C}_{22} \mathrm{H}_{27} \mathrm{O}_{6} \mathrm{P}$ (418.2). Calculated, \%: C, 63.15; H, 6.50.

\section{Diethyl 2-phenylethyloxy(3-methoxy-4-propargyloxyphenyl) methyl phosphonate (8f)}

$0.35 \mathrm{~g}$, yield $82 \%$ colorless oil. ${ }^{1} \mathrm{H}$ NMR spectrum, $(300 \mathrm{MHz}$, $\left.\mathrm{CDCl}_{3}\right), \delta, \operatorname{ppm}(J, \mathrm{~Hz}): 1.16-1.24\left(\mathrm{~m}, 6 \mathrm{H}, 2 \mathrm{CH}_{3}\right), 2.51(\mathrm{~s}, 1 \mathrm{H}, \mathrm{CH})$, 2.88-2.93 (m, 2H, $\left.\mathrm{CH}_{2}\right), 3.67-3.71\left(\mathrm{~m}, 2 \mathrm{H}, \mathrm{CH}_{2}\right), 3.79(\mathrm{~s}, 3 \mathrm{H}$, $\left.\mathrm{OCH}_{3}\right), 3.92-4.04\left(\mathrm{~m}, 4 \mathrm{H}, 2 \mathrm{CH}_{2}\right), 4.56\left(\mathrm{~d}, J_{\mathrm{HP}}=15.0 \mathrm{~Hz}, 1 \mathrm{H}\right.$, CHP), 4.73 (d, $J=3.0 \mathrm{~Hz}, 2 \mathrm{H}, \mathrm{CH}_{2}$ ), 6.86-6.98 (m, 3H, H-Ar), 7.17-7.38 (m, 5H, H-Ar). ${ }^{13} \mathrm{C}-\mathrm{NMR}$ (75.0 $\mathrm{MHz} \mathrm{CDCl}_{3}$ ), $\delta$, ppm: $16.3\left(\mathrm{~d}, J_{\mathrm{CP}}=6.0, \mathrm{CH}_{3}\right), 16.4\left(\mathrm{~d}, J_{\mathrm{CP}}=6.0, \mathrm{CH}_{3}\right), 36.2\left(\mathrm{CH}_{2}\right), 55.8$ $\left(\mathrm{OCH}_{3}\right), 56.8\left(\mathrm{OCH}_{2}\right), 62.9\left(\mathrm{~d}, J_{\mathrm{CP}}=6.8, \mathrm{CH}_{2} \mathrm{OP}\right), 63.2\left(\mathrm{~d}, J_{\mathrm{CP}}=\right.$ 6.8, $\mathrm{CH}_{2} \mathrm{OP}$ ), 71.5 (d, $\left.J_{\mathrm{CP}}=13.6, \mathrm{CH}_{2} \mathrm{OCHP}\right), 75.8(\mathrm{C} \equiv \mathrm{CH}), 78.5$ $(\mathrm{C} \equiv \mathrm{CH}), 78.6\left(\mathrm{~d}, J_{\mathrm{CP}}=169.9, \mathrm{CHP}\right), 111.2,111.3$, 113.9, 120.5, 120.6, 126.2 , 128.3, 128.6, 129.0, 138.7, 146.9, 149.7 (C-Ar); ${ }^{31} \mathrm{P}-$
NMR (194.4 MHz, $\mathrm{CDCl}_{3}$ ), $\delta$, ppm: 18.9 (P). Found, \%: C, 63.69; $\mathrm{H}$, 6.72. For $\mathrm{C}_{23} \mathrm{H}_{29} \mathrm{O}_{6} \mathrm{P}$ (432.2). Calculated, \%: C, 63.88; H, 6.76.

\section{Diethyl((5-methyl-2-phenyl-1,3-dioxan-5-yl)methoxy)(4- methoxyphenyl)methyl phosphonate (10a)}

$0.29 \mathrm{~g}$, yield $62 \%$ colorless oil. ${ }^{1} \mathrm{H}$ NMR spectrum, $(300 \mathrm{MHz}$, $\left.\mathrm{CDCl}_{3}\right), \delta, \operatorname{ppm}(J, \mathrm{~Hz}): 0.87\left(\mathrm{~s}, 3 \mathrm{H}, \mathrm{CH}_{3}\right), 1.20-1.33(\mathrm{~m}, 6 \mathrm{H}$, $\left.2 \mathrm{CH}_{3}\right), 3.59-3.73\left(\mathrm{~m}, 4 \mathrm{H}, 2 \mathrm{CH}_{2}\right), 3.81\left(\mathrm{~s}, 3 \mathrm{H}, \mathrm{OCH}_{3}\right), 3.88-4.16$ $\left(\mathrm{m}, 6 \mathrm{H}, 3 \mathrm{CH}_{2}\right), 4.65\left(\mathrm{~d}, J_{\mathrm{HP}}=15.0 \mathrm{~Hz}, 1 \mathrm{H}, \mathrm{CHP}\right), 5.39(\mathrm{~s}, \mathrm{IH}, \mathrm{CH})$, 6.90 (d, $J=9.0 \mathrm{~Hz}, 2 \mathrm{H}, \mathrm{H}-\mathrm{Ar}), 7.32-7.43$ (m, 7H, H-Ar). ${ }^{13} \mathrm{C}-\mathrm{NMR}$ (75.0 MHz, $\left.\mathrm{CDCl}_{3}\right), \delta$, ppm: $16.4\left(\mathrm{~d}, J_{\mathrm{CP}}=5.3, \mathrm{CH}_{3}\right), 16.5\left(\mathrm{~d}, J_{\mathrm{CP}}=\right.$ 5.3, $\left.\mathrm{CH}_{3}\right), 19.2\left(\mathrm{CH}_{3}\right), 34.6(\mathrm{C}), 55.7\left(\mathrm{OCH}_{3}\right), 62.6\left(\mathrm{~d}, J_{\mathrm{CP}}=7.8\right.$, $\left.\mathrm{CH}_{2} \mathrm{OP}\right), 63.1\left(\mathrm{~d}, J_{\mathrm{CP}}=7.8, \mathrm{CH}_{2} \mathrm{OP}\right), 72.8\left(\mathrm{OCH}_{2}\right), 73.0\left(\mathrm{~d}, J_{\mathrm{CP}}=\right.$ 14.3, $\left.\mathrm{CH}_{2} \mathrm{OCHP}\right), 73.7\left(\mathrm{OCH}_{2}\right), 79.0\left(\mathrm{~d}, J_{\mathrm{CP}}=170.6, \mathrm{CHP}\right), 101.7$ $(\mathrm{OCH}), 113.9,114.0,126.1,126.2,128.2$, 128.9, 129.5, 129.6, 138.3, 159.8 (C-Ar). ${ }^{31} \mathrm{P}-\mathrm{NMR}$ (194.4 $\mathrm{MHz} \mathrm{CDCl}_{3}$ ), $\delta$, ppm: 19.2 (P). Found, \%: C, 61.95; H, 7.14. For $\mathrm{C}_{24} \mathrm{H}_{33} \mathrm{O}_{7} \mathrm{P}$ (464.2). Calculated, \%: C, 62.06; H, 7.16.

\section{Diethyl((5-methyl-2-phenyl-1,3-dioxan-5-yl)methoxy)(3,4- dimethoxyphenyl)-methyl phosphonate (10b)}

$0.35 \mathrm{~g}$, yield $70 \%$ colorless oil. ${ }^{1} \mathrm{H}$ NMR spectrum, $(300 \mathrm{MHz}$, $\left.\mathrm{CDCl}_{3}\right), \delta, \operatorname{ppm}(J, \mathrm{~Hz}): 0.87\left(\mathrm{~s}, 3 \mathrm{H}, \mathrm{CH}_{3}\right), 1.19(\mathrm{t}, J=6.0 \mathrm{~Hz}, 3 \mathrm{H}$, $\left.\mathrm{CH}_{3}\right), 1.26\left(\mathrm{t}, J=6.0 \mathrm{~Hz}, 3 \mathrm{H}, \mathrm{CH}_{3}\right), 3.63-3.75\left(\mathrm{~m}, 4 \mathrm{H}, 2 \mathrm{CH}_{2}\right)$, 3.59-3.79 (m, 2H, $\left.\mathrm{CH}_{2}\right), 3.88\left(\mathrm{~s}, 3 \mathrm{H}, \mathrm{OCH}_{3}\right), 3.96\left(\mathrm{~s}, 3 \mathrm{H}, \mathrm{OCH}_{3}\right)$, 4.01-4.15 (m, $\left.4 \mathrm{H}, 2 \mathrm{CH}_{2}\right), 4.63\left(\mathrm{~d}, J_{\mathrm{HP}}=15.0 \mathrm{~Hz}, 1 \mathrm{H}, \mathrm{CHP}\right), 5.38$ (s, IH, CH), 6.84-7.00 (m, 2H, H-Ar), 7.05 (s, 1H, H-Ar), 7.28-7.37 (m, 5H, H-Ar). ${ }^{13} \mathrm{C}-\mathrm{NMR}$ (75.0 MHz, $\mathrm{CDCl}_{3}$ ), $\delta$, ppm: 16.4 (d, $J_{\mathrm{CP}}$ $\left.=6.0, \mathrm{CH}_{3}\right), 16.5\left(\mathrm{~d}, J_{\mathrm{CP}}=6.0, \mathrm{CH}_{3}\right), 19.1\left(\mathrm{CH}_{3}\right), 35.0(\mathrm{C}), 55.8$ $\left(\mathrm{OCH}_{3}\right), 55.9\left(\mathrm{OCH}_{3}\right), 62.8\left(\mathrm{~d}, J_{\mathrm{CP}}=7.8, \mathrm{CH}_{2} \mathrm{OP}\right), 63.1\left(\mathrm{~d}, J_{\mathrm{CP}}=\right.$ $7.8 \mathrm{CH}_{2} \mathrm{OP}$ ), 72.9 (d, $\left.J_{\mathrm{CP}}=14.3, \mathrm{CH}_{2} \mathrm{OCHP}\right), 73.2\left(\mathrm{OCH}_{2}\right), 73.3$ $\left(\mathrm{OCH}_{2}\right), 79.0\left(\mathrm{~d}, J_{\mathrm{CP}}=170.6, \mathrm{CHP}\right), 101.8(\mathrm{OCH}), 110.8,111.1$, 111.2, 120.8, 120.9, 126.1, 128.2, 128.3, 128.9, 138.3, 149.0, 149.3 (C-Ar). ${ }^{31} \mathrm{P}-\mathrm{NMR}\left(194.4 \mathrm{MHz}, \mathrm{CDCl}_{3}\right.$ ), $\delta$, ppm: 19.2 (P). Found, \%: C, 60.62; H, 6.85. For $\mathrm{C}_{25} \mathrm{H}_{35} \mathrm{O}_{8} \mathrm{P}$ (494.2). Calculated, \%: C, 60.72; $\mathrm{H}, 7.13$.

\section{Diethyl 2-( $N$-methyl- $N$-phenylamino)ethyloxy(4-} methoxyphenyl)methyl phosphonate (12)

$0.12 \mathrm{~g}$, yield $29 \%$ colorless oil. ${ }^{1} \mathrm{H}$ NMR spectrum, $(300 \mathrm{MHz}$, $\left.\mathrm{CDCl}_{3}\right), \delta, \operatorname{ppm}(J, \mathrm{~Hz}): 1.21\left(\mathrm{t}, J=6.0 \mathrm{~Hz}, 3 \mathrm{H}, \mathrm{CH}_{3}\right), 1.26(\mathrm{t}, J=$ $\left.6.0 \mathrm{~Hz}, 3 \mathrm{H}, \mathrm{CH}_{3}\right), 2.98\left(\mathrm{~s}, 3 \mathrm{H}, \mathrm{CH}_{3}\right), 3.57-3.68\left(\mathrm{~m}, 4 \mathrm{H}, 2 \mathrm{CH}_{2}\right)$, $3.82\left(\mathrm{~s}, 3 \mathrm{H}, \mathrm{OCH}_{3}\right), 3.93-4.11\left(\mathrm{~m}, 4 \mathrm{H}, 2 \mathrm{CH}_{2}\right), 4.58\left(\mathrm{~d}, J_{\mathrm{HP}}=\right.$ $15.0 \mathrm{~Hz}, 1 \mathrm{H}, \mathrm{CHP}), 6.68$ (d, $J=9.0 \mathrm{~Hz}, 2 \mathrm{H}, \mathrm{H}-\mathrm{Ar}), 6.87$ (d, $J=$ $9.0 \mathrm{~Hz}, 2 \mathrm{H}, \mathrm{H}-\mathrm{Ar}), 7.18-7.35$ (m, 5H, H-Ar). ${ }^{13} \mathrm{C}-\mathrm{NMR}(75.0 \mathrm{MHz}$, $\left.\mathrm{CDCl}_{3}\right), \delta$, ppm: $16.3\left(\mathrm{~d}, J_{\mathrm{CP}}=6.0, \mathrm{CH}_{3}\right), 16.4\left(\mathrm{~d}, J_{\mathrm{CP}}=6.0, \mathrm{CH}_{3}\right)$, $36.2\left(\mathrm{CH}_{3}\right), 55.7\left(\mathrm{OCH}_{3}\right), 55.8\left(\mathrm{NCH}_{2}\right), 62.8\left(\mathrm{~d}, J_{\mathrm{CP}}=7.8, \mathrm{CH}_{2} \mathrm{OP}\right)$, $63.6\left(\mathrm{~d}, J_{\mathrm{CP}}=7.8, \mathrm{CH}_{2} \mathrm{OP}\right), 71.4\left(\mathrm{~d}, J_{\mathrm{CP}}=14.3, \mathrm{CH}_{2} \mathrm{OCHP}\right), 78.7$ $\left(\mathrm{d}, J_{\mathrm{CP}}=163.1, \mathrm{CHP}\right), 110.7,110.8,110.9,120.7,120.8,126.1$, 127.1, 128.2, 128.9, 138.7, 149.0 (C-Ar). ${ }^{31} \mathrm{P}-\mathrm{NMR}$ (194.4 MHz, $\left.\mathrm{CDCl}_{3}\right) \delta$, ppm: 19.1 (P). Found, \%: C, 61.87; H, 7.38; N, 3.44. For $\mathrm{C}_{21} \mathrm{H}_{30} \mathrm{NO}_{5} \mathrm{P}$ (407.2). Calculated, \%: C, 61.90; H, 7.42; N, 3.44.

\section{Conflicts of interest}

There are no conflicts to declare. 


\section{References}

1 R. Engel, Chem. Rev., 1977, 77, 349-367.

2 R. L. Hilderbrand, The Role of Phosphonates in Living Systems, CRC press, Boca Raton, FL, 1983.

3 D. J. Schauer and P. Helquist, Synthesis, 2006, 3654-3660.

4 D. C. Reuter, J. E. McIntosh, A. C. Guinn and A. M. Madera, Synthesis, 2003, 2321-2324.

5 U. S. Dakarapu, A. Bokka, P. Asgari, G. Trog, Y. Hua, H. H. Nguyen, N. Rahman and J. Jeon, Org. Lett., 2015, 17, 5792-5795.

6 A. K. Bhattacharya and G. Thyagarajan, Chem. Rev., 1981, 81, 415-430.

7 B. A. Arbuzov, Pure Appl. Chem., 1964, 9, 307-336.

8 Y. Belabassi, S. Alzghari and J. L. Montchamp, J. Organomet. Chem., 2008, 693, 3171-3178.

9 R. A. Dhokale and S. B. Mhaske, Org. Lett., 2013, 15, 22182221.

10 H. Firouzabadi, N. Iranpoor, S. Sobhani and Z. Amoozgar, Synthesis, 2004, 1771-1774.

11 B. Kaboudin, Tetrahedron Lett., 2003, 44, 1051-1053.

12 R. L. Hilderbrand, The Role of Phosphonates in Living Systems, CRC press, Boca Raton, FL, 1983.

13 R. U. Pokalwar, R. V. Hangarge, P. V. Maske and M. S. Shingare, ARKIVOC, 2006, xi, 196-204.
14 D. Q. Shi, Z. L. Sheng, X. P. Liu and H. Wu, Heteroat. Chem., 2003, 14, 266-268.

15 A. J. Ganzhorn, J. Hoflack, P. D. Pelton, F. Strasser, M. C. Chanal and S. R. Piettre, Bioorg. Med. Chem., 1998, 6, 1865-1874.

16 J. Guin, Q. Wang, M. Gemmeren and B. List, Angew. Chem., Int. Ed., 2015, 54, 355-358.

17 H. I. Hansen and J. Kehler, Synthesis, 1999, 1925-1927.

18 R. R. Schmidt and W. Kinzy, Adv. Carbohydr. Chem. Biochem., 1994, 50, 21-123.

19 R. R. Schmidt and K.-H. Jung, in Preparative Carbohydrate Chemistry, ed. S. Hannessian, Marcel Dekker, Inc., New York, 1997, pp. 283-312.

20 I. A. I. Ali and W. Fathalla, ARKIVOC, 2009, xiii, 193-199.

21 W. Fathalla, Sci. J. Chem., 2014, 2, 38-43.

22 I. A. I. Ali and W. Fathalla, Curr. Org. Chem., 2013, 17, 19031909.

23 D. Rhodes and R. F. Newton, US Pat. 4085222 A, US 05/ $523,238,1978$.

24 L. Gavara, T. Boisse, J.-P. Hénichart, A. Daïch, B. Rigo and P. Gautret, Tetrahedron, 2010, 66, 7544-7561.

25 J. Manfred, A. Dieter, L. Reinhard, F. Rainer, R. HansJochem, S. Rolf and H. Horst, US Pat. 4327025 A1, Bayer Aktiengesellschaft, 1982. 